Original article

\title{
The peroxisomal fatty acid transporter ABCD1/PMP-4 is required in the $C$. elegans hypodermis for axonal maintenance: A worm model for adrenoleukodystrophy
}

\author{
Andrea Coppa $^{\mathrm{a}}$, Sanjib Guha ${ }^{\mathrm{a}}$, Stéphane Fourcade ${ }^{\mathrm{a}, \mathrm{b}}$, Janani Parameswaran ${ }^{\mathrm{a}, \mathrm{b}}$, \\ Montserrat Ruiz ${ }^{\mathrm{a}, \mathrm{b}}$, Ann B. Moser ${ }^{\mathrm{c}}$, Agatha Schlüter ${ }^{\mathrm{a}, \mathrm{b}}$, Michael P. Murphy ${ }^{\mathrm{d}}$, Jose Miguel Lizcano ${ }^{\mathrm{e}}$, \\ Antonio Miranda-Vizuete ${ }^{\mathrm{f}}$, Esther Dalfó ${ }^{\mathrm{e}, \mathrm{g}, * *}$, Aurora Pujol ${ }^{\mathrm{a}, \mathrm{b}, \mathrm{h}, *}$ \\ ${ }^{a}$ Neurometabolic Diseases Laboratory, Institut d'Investigació Biomèdica de Bellvitge (IDIBELL), Hospital Duran i Reynals, L'Hospitalet de Llobregat, Spain \\ ${ }^{\mathrm{b}}$ CIBERER U759, Center for Biomedical Research on Rare Diseases, Spain \\ ${ }^{c}$ Peroxisomal Diseases Laboratory, Kennedy Krieger Institute, 707 N. Broadway, Baltimore, MD, 21205, USA \\ ${ }^{\mathrm{d}}$ MRC Mitochondrial Biology Unit, Cambridge, UK \\ ${ }^{\mathrm{e}}$ Departament de Bioquímica i Biologia Molecular, Institut de Neurociències, Facultat de Medicina, Universitat Autònoma de Barcelona, 08193, Bellaterra (Barcelona), \\ Spain \\ ${ }_{\mathrm{f}}^{\mathrm{f}}$ Instituto de Biomedicina de Sevilla (IBIS), Hospital Universitario Virgen del Rocío /CSIC/ Universidad de Sevilla, E-41013, Sevilla, Spain \\ ${ }^{\mathrm{g}}$ Faculty of Medicine, University of Vic-Central University of Catalonia (UVic-UCC), 08500, Vic, Spain \\ ${ }^{\mathrm{h}}$ ICREA (Institució Catalana de Recerca i Estudis Avançats), Barcelona, Spain
}

\section{A R T I C L E I N F O}

\section{Keywords:}

$\mathrm{X}$-linked adrenoleukodystrophy

Axonal degeneration

Hypodermis

Mitochondria redox imbalance

Lipid droplets

Peroxisomes

\begin{abstract}
A B S T R A C T
Adrenoleukodystrophy is a neurometabolic disorder caused by a defective peroxisomal ABCD1 transporter of very long-chain fatty acids (VLCFAs). Its pathogenesis is incompletely understood. Here we characterize a nematode model of X-ALD with loss of the pmp-4 gene, the worm orthologue of $A B C D 1$. These mutants recapitulate the hallmarks of X-ALD: i) VLCFAs accumulation and impaired mitochondrial redox homeostasis and ii) axonal damage coupled to locomotor dysfunction. Furthermore, we identify a novel role for PMP-4 in modulating lipid droplet dynamics. Importantly, we show that the mitochondria targeted antioxidant MitoQ normalizes lipid droplets size, and prevents axonal degeneration and locomotor disability, highlighting its therapeutic potential. Moreover, PMP-4 acting solely in the hypodermis rescues axonal and locomotion abnormalities, suggesting a myelin-like role for the hypodermis in providing essential peroxisomal functions for the nematode nervous system.
\end{abstract}

\section{Introduction}

Peroxisomes are single membrane-bound ubiquitous organelles that play key roles in redox homeostasis and the metabolism of lipids, in particular fatty acid $\beta$-oxidation, ether phospholipids, and bile acids [1]. Impairments in any of these essential pathways are associated with major clinical signs and symptoms, usually involving the nervous system [2]. X-linked adrenoleukodystrophy (X-ALD, McKusick no. 300100) is the most common peroxisomal disease and leukodystrophy with an incidence of 1:14700 births [3]. X-ALD is caused by a loss of function of the $A B C D 1$ gene, which encodes a peroxisomal transporter that imports very long-chain fatty acids (VLCFAs) to be $\beta$-oxidized [4].
As a consequence, VLCFAs, especially hexacosanoic acid or C26:0, accumulate in tissues and plasma and constitute a pathognomonic biomarker for diagnosis. X-ALD is a complex inherited syndrome in which the same mutation in the $A B C D 1$ gene can lead to highly divergent clinical phenotypes, such as childhood cerebral adrenoleukodystrophy (cALD), chronic progressive adult-onset adrenomyeloneuropathy (AMN) or cerebral AMN (cAMN) [5,6]. Therapeutic options are scarce, and when diagnosed early, the cerebral forms of the disease (cALD and cAMN) are only adequately treatable with an allogeneic bone marrow transplant $[7,8]$ or recently, with haematopoietic stem cell gene therapy for cALD $[9,10]$. However, no pharmacological treatment has been shown to be beneficial for either form of the disease [11],

\footnotetext{
* Corresponding author. Neurometabolic Diseases Laboratory; IDIBELL; Gran Via, 199; 08908, L'Hospitalet de Llobregat; Barcelona, Spain.

${ }^{* *}$ Corresponding author. Department of Biochemistry and Molecular Biology, Institut de Neurociències, Faculty of Medicine, M2, Universitat Autònoma de Barcelona (UAB), Bellaterra Campus, 08193, Cerdanyola del Vallés; Spain.

E-mail addresses: esther.dalfo@umedicina.cat (E. Dalfó), apujol@idibell.cat (A. Pujol).
} 
although several repurposed drugs have been proposed [12-15].

The two mouse models of X-ALD ( $A b c d 1^{-}$and $A b c d 1^{-} / A b c d 2^{-/-}$ mice) develop late-onset axonopathy, with signs and symptoms resembling AMN visible at 20 and 12 months of age, respectively [16,17]. Using these mouse models and patient samples, several studies have indicated that VLCFA-induced oxidative stress is a critical, early pathogenic factor in X-ALD [18-20], although the exact mechanisms by which redox imbalance causes neurodegeneration in X-ALD are incompletely understood.

Here, we established a cost-effective disease model with the aim of identifying critical steps leading to axonal demise and establishing a rapid and amenable platform for high-throughput drug screening in the nematode Caenorhabditis elegans. pmp-4 is the worm orthologue of $A B C D 1$, and its function has thus far been unexplored. Despite the fact that the C. elegans nervous system is not myelinated [21], thus precluding the study of the physiopathology of the infantile form of X-ALD (cALD), this work indicates that pmp-4(ok396) worms may constitute a valuable model of the axonopathy occurring in the adult form of the disease, AMN. This study sheds light on the mechanisms leading to mitochondrial and lipid droplet (LD) metabolism impairment while highlighting the prominent role of the hypodermis in axonal maintenance in the nematode.

\section{Material and methods}

A more detailed explanation of the methodology, strains, and plasmid information is provided in the Supplementary Methodology section.

\subsection{Products}

MitoQ (mitoquinone mesylate:10-(4,5-dimethoxy-2-methyl-3,6dioxo-1,4 cyclohexadienyl) decyltriphenyl-phosphonium methanesulfonate) was provided by MP Murphy. CoQ10, paraquat (PQT), 2,4Dinitrophenylhydrazine, thenoyltrifluoroacetone (TTFA), antimycin, sodium azide and dimethylsulfoxide (DMSO) were purchased from Sigma-Aldrich. $\mathrm{H}_{2}$ DCFDA and MitoSOX Red were obtained from Life Technologies.

\subsection{Strains}

Worm strains were maintained under standard conditions on Escherichia coli OP50 at $20{ }^{\circ} \mathrm{C}$. All the strains used were provided by the Caenorhabditis Genetic Center (CGC) unless otherwise indicated. N2 (Bristol) was used as the WT strain (N2). The partial deletion strain RB675, pmp-4(ok396), was backcrossed 10 times into the N2 strain prior to experimental work.

All the strains mentioned in the manuscript are detailed in Supplementary Table S1. Plasmid HYM772 (for targeting peroxisomes in hypodermis) was kindly provided by Dr. Ho Yi Mak (Stowers Institute for Medical Research, Kansas City, MO, USA).

\subsection{In silico sequence analysis}

The alignment of protein sequences between the indicated species was performed by ClustalW software, version 2 [22]. The Pex19 binding site was predicted by using the PTS predictor tool in the peroxisome database: www.peroxisomedb.org.

\subsection{Plasmids and nematode transgenesis}

The vectors pPD95.79, pPD95.77 and pPD96.32 (Addgene, Teddington, UK) were used as backbones for all the plasmids generated in this study. The constructs are listed in Supplementary Table S2, and detailed cloning information will be provided upon request. Transgenic strains were generated using standard microinjection techniques [23] and are listed in Table S1. Plasmids were injected into worm adult gonads alone or together with the plasmid pRF4 (100 ng/ $\mu$ l), which encodes a mutant collagen [rol-6(su1006)] that induces a dominant "roller" phenotype or the plasmid pCFJ90 $(2.5 \mathrm{ng} / \mu \mathrm{l})$, which expresses mCherry under the control of the myo-2 promoter [Pmyo-2::mCherry::unc-54utr], as co-injection markers depending on the experiments. In all cases, at least 3 independent transgenic lines were generated and analysed, all showing similar results.

\subsection{Generation of the PMP-4 antibody}

A PMP-4 antibody was obtained following the standard procedures at the IGBMC core facilities, Strasbourg, France. The PMP-4 peptide CQLLGGNEDHLNMTIDTDDSE (see Supplementary Fig. S1A) was coupled with maleimide-activated ovalbumin (Thermo Scientific, Madrid, Spain) and injected into rabbits. The polyclonal anti-PMP-4 antibody was purified from rabbit serum through a gel matrix (Sulfolink * coupling gel). Fractions of $1 \mathrm{ml}$ were eluted from the column, tested for activity by immunoblotting, aliquoted and stored at $-20{ }^{\circ} \mathrm{C}$ until use.

\subsection{Analysis of PMP-4 expression by western blotting}

PMP-4 expression was performed in synchronized populations of worms obtained by bleaching. To collect L1 worms, eggs were allowed to hatch on Nematode growth medium (NGM)-seeded plates and collected after $4-6 \mathrm{~h}$ of feeding at $20{ }^{\circ} \mathrm{C}$. To isolate the other larval stages, the eggs were allowed to hatch on $\mathrm{M} 9 \mathrm{O} / \mathrm{N}$ at $20^{\circ} \mathrm{C}$, and the resulting synchronized L1 worms were added to NGM-seeded plates; the animals were harvested at the appropriate developmental stage after $10 \mathrm{~h}$ (for L2 animals), $24 \mathrm{~h}$ (for L3 animals), $48 \mathrm{~h}$ (for L4 animals), and 7 days (for post-reproductive adult animals). The worms were washed extensively with M9 to remove bacteria, and the worm pellets were frozen at $-80{ }^{\circ} \mathrm{C}$.

The Western blot analysis was performed as described [15]. Eight micrograms of protein was separated by $8 \%$ SDS-polyacrylamide gel electrophoresis (SDS-PAGE) and blotted onto nitrocellulose membranes (Bio-Rad, Barcelona, Spain). The membranes were then incubated with anti-PMP-4 (1:500 rabbit polyclonal) and anti-rabbit secondary antibodies (1:5000. DAKO, Barcelona, Spain), and the proteins were visualized with an ECL plus detection reagent (GE Healthcare, Barcelona, Spain). The same process was performed for the detection of DNP-derived oxidative damage with slight modifications specific for the detection of oxidized proteins and detailed below in the oxidative stress section. Densitometric analysis was performed using ImageJ software.

\subsection{Immunocytochemistry}

Whole-mount immunofluorescent staining for PMP-4 and/or GFP was performed as described [24]. The samples were probed with primary antibodies against PMP-4 described in the previous section at a 1:100 dilution and/or anti-GFP (Abcam, Cambridge, UK) at a 1:200 dilution. Secondary antibodies conjugated to Alexa Fluor 555 (PMP-4) dye and/or Alexa Fluor 555 (GFP) (Molecular Probes, Barcelona, Spain) were used at a 1:1000 dilution. Images were acquired on a Leica Confocal SP5 microscope. Nuclei were detected by DAPI counterstaining.

\subsection{Imaging}

Protein blot images were acquired using the Fujifilm Image Reader (NIH, ver. 1.43). The quantification of protein levels was performed using Multigage software v3.0 (Fujifilm).

Fluorescent microscopy was performed using a Nikon Eclipse E800 epifluorescence microscope equipped with an Endow GFP HYQ filter cube (Chroma Technology). Confocal microscopy was performed using a Leica TCS-SP5 confocal spectral microscope (Barcelona, Spain) and analysed by ImageJ software (NIH, ver. 1.43). Video recordings of the 
thrashing behaviour were performed on a stereomicroscope (SteREO Lumar. V12, Carl Zeiss, Madrid, Spain) coupled to a digital camera (Jenoptik, model ProgRes CF scan).

\subsection{Lipid extraction and fatty acid quantification}

Newly hatched L1 larvae were grown on regular NGM plus OP50 for $48 \mathrm{~h}$ at $20{ }^{\circ} \mathrm{C}$. L4 staged animals were washed and collected from 10 plates of $10 \mathrm{~cm}$ in diameter and cleaned from bacteria with M9 buffer. Subsequently, the worms were washed twice with $\mathrm{S}$ Basal buffer and finally with Optimal LC/MS $\mathrm{H}_{2} \mathrm{O}$. The worms were settled by gravity on ice, and the water was carefully removed. The worm pellets were quickly frozen in liquid nitrogen and stored frozen at $-80{ }^{\circ} \mathrm{C}$ until analysis.

For homogenization, the frozen cell pellets were briefly thawed, and $0.5 \mathrm{ml}$ MilliQ water was added, mixed by vortexing and transferred to a "ball sonicator tube". The original tube was rinsed with $0.5 \mathrm{ml}$ MilliQ water, and the rinse was also transferred to the "ball sonicator tube". A small steel ball was added, and the tube was capped. The tubes were mixed in the cold for 2-3 min 25 times per $1 \mathrm{~s}$ in the Qiagen TissueLyser II. The samples appeared to be well mixed and broken into fine particles. Five-microliter aliquots were removed for total protein, $10-\mu \mathrm{l}$ aliquots were removed for LC/MS/MS, and $0.5 \mathrm{ml}$ x 2 aliquots were removed for total lipid extract by the Folch method [25]. The Lyso-PC FIA-MS/MS analysis was performed as previously shown [26] (WT, $\mathrm{n}=12$ and pmp-4(ok396), $\mathrm{n}=10$ ). The daf-22(ok693) strain accumulates VLCFA [27] and was therefore used as a positive control $(n=6)$. The results are expressed as $\mathrm{pmol} / \mathrm{mg}$ protein.

\subsection{Thrashing assay}

Thrashing is a rhythmic pattern of activity where the worm oscillates side-to-side around its midpoint. A single thrash was defined by a movement through the midpoint and back. The thrashing assay was performed blindly and in liquid medium as described previously [28]. A drop of $2 \%$ agarose was poured over the glass slide and allowed to dry. After adding $20 \mu \mathrm{l}$ of M9, L4 or L4 + 7 synchronized animals, the worms were placed on the drop and left for $2 \mathrm{~min}$. Thrashes were counted for $30 \mathrm{~s}$, and every animal was counted three consecutive times to obtain an average. This value was multiplied by two to obtain an estimate per minute. A single thrash was defined as a complete change in the direction of the body down the midline. Animals who were motionless for $10 \mathrm{~s}$ were discarded from the analysis.

\subsection{Quantification of GABAergic-associated abnormalities using the juIs76 [Punc-25::gfp] reporter strain}

Worms were grown at $20{ }^{\circ} \mathrm{C}$ for at least two generations before the experiments. Animal age refers to the adult age measured in days, calculated by adding one day consecutively from the given animal went through L4 [29]. The animals were transferred daily to avoid mixing populations, and the animals were considered dead when they stopped pharyngeal pumping and failed to respond to mild touch by a platinum wire picker. After synchronization, F1 hermaphrodite animals were maintained at $20{ }^{\circ} \mathrm{C}$ until the late $\mathrm{L} 4$ larval stage and then transferred to the final assay plates (50 worms/plate) supplemented or not with the indicated concentration of drugs (PQT $0.2 \mathrm{mM}$, CoQ $1 \mathrm{mg} / \mathrm{ml}$ and MitoQ, $5 \mu \mathrm{g} / \mathrm{ml}$ ) when required.

On the actual day of the experiment, worms of the appropriate age and genotype, with or without drugs, were washed, anaesthetized in $10 \mathrm{mM}$ sodium azide on $6 \%$ agar pads and subsequently mounted for image analysis. The worms were scored for the number of GABAergic neurons, gaps in the ventral cord, gaps in the dorsal cord and defects in axonal morphology [30].

The applied formula used to quantify axonal damage is: \% of axonal damage $=[($ (number of commissural abnormalities + number of gaps $/$ animal))/number of axons detected) x100].

The experiment was performed blindly and by different investigators to guarantee the reliability of the obtained measurements.

\subsection{Fasting experiments}

L4 animals were starved for $24 \mathrm{~h}$ under standard conditions at $20^{\circ} \mathrm{C}$.

\subsection{Analysis of lipid droplets}

Fat accumulation was analysed by fixative and non-fixative live methodology. The animals were washed with M9 buffer and fixed with $2 \%$ paraformaldehyde. The worms were then subjected to three freezethaw cycles to disrupt the cuticles and incubated for $5 \mathrm{~min}$ and $10 \mathrm{~min}$ on ice. After 3 washes with cold M9, worms were dehydrated through an ethanol series $(25 \%, 50 \%, 70 \%)$ and $2-5$ volumes of $3 \%$ Sudan Black B solution (Sigma-Aldrich, Madrid, Spain) were added to the worms, which were then incubated overnight. The worms were rehydrated through an ethanol series $(70 \%, 50 \%, 25 \%)$ and finally with M9. Lipid droplets were detectable as large black granules under a bright-field microscope.

The non-fixative live methodology was performed by using the strain VS20 (hjIs67 [Patgl-1::atgl-1::gfp]), in which ATGL-1 localizes to the surface of the lipid droplets [31]. This strain was crossed with pmp4(ok396) animals (pmp-4(ok396); hjIs67 [Patgl-1::atgl-1::gfp]), and the resulting progeny were analysed by confocal microscopy in parallel with the corresponding age-matched controls.

\subsection{Oxidative stress assays}

The sensitivity to oxidative stress was analysed systematically by using different approaches.

Protein carbonylation: Synchronized L4 animals grown at $20{ }^{\circ} \mathrm{C}$ were used. Fifteen micrograms of worm proteins were run on an $8 \%$ SDS gel, transferred to nitrocellulose and incubated with a solution of $0.2 \%$ 2,4-Dinitrophenylhydrazine (DNPH, Sigma-Aldrich, Madrid, Spain). This method is based on the formation of a hydrazone (DNP) resulting from the reaction of protein-bound carbonyl and 2,4-DNPH [20]. An antibody against DNP was used to detect carbonylated protein. Carbonylated proteins were finally detected with ECL western blotting analysis systems described previously for PMP-4 Western blot detection.

Measurement of intracellular ROS was performed as previously described [32,33]. The amount of ROS was detected with the membrane-permeable non-fluorescent dye 2,7-dichlorodihydrofluoresceindiacetate $\left(\mathrm{H}_{2} \mathrm{DCFDA}\right)$, which can enter the cell where it is converted to a polar non-fluorescent derivative (dichlorofluorescein diacetate, $\mathrm{H}_{2}$ DCFDA). Then, $\mathrm{H}_{2}$ DCFDA is rapidly oxidized to the highly fluorescent $2^{\prime}, 7^{\prime}$-dichlorofluorescein in the presence of intracellular ROS. To detect changes in the levels of ROS, a stock solution of $10 \mathrm{mM}$ carboxy$\mathrm{H}_{2}$ DCFDA (Molecular Probes, Barcelona, Spain) in DMSO was diluted to $10 \mu \mathrm{M}$ in $\mathrm{M} 9$ buffer. Young adult worms were transferred into the staining solution and stained for $30 \mathrm{~min}$ at $20{ }^{\circ} \mathrm{C}$. The worms were mounted on a thick layer of half-dried agar pad on microscopic glass slides and then subjected to fluorescence microscopy (see above).

Measurement of mitochondrial ROS with MitoSOx probes was performed as previously described [34]. MitoSOX Red (Life Technologies, Madrid, Spain) was diluted in DMSO at $10 \mathrm{mM}$ and frozen at $-20{ }^{\circ} \mathrm{C}$ as stock. Before staining, the stocks were diluted in M9 buffer at a 1:1000 dilution. L4-staged worms were transferred into the staining solution and stained overnight at $20{ }^{\circ} \mathrm{C}$ in a mixer. After incubation, the worms were washed twice with M9 buffer and mounted on a $6 \%$ layer of halfdried agar pad on microscopic glass slides and then subjected to fluorescence microscopy. Pictures were taken by NIS software, and the fluorescence was analysed by ImageJ software.

Sensitivity to mitochondrial inhibitors: L4 synchronized animals 
were incubated separately with PQT (4 mM) [35], thenoyltrifluoroacetone (TTFA) (2 mM), 3-nitropropionic acid (3-NP) (100 mM), antimycin $(1.27 \mathrm{mM})$ or sodium azide $(1 \mathrm{mM})$ [36]. The worms were considered dead when they failed to move, either spontaneously or in response to touch.

\subsection{Antioxidant treatments}

\subsubsection{CoQ assay}

CoQ nematode treatment was performed as previously described [30]. CoQ10 (100 mg) was dissolved in $10 \mathrm{ml}$ of distilled water containing $0.06 \%$ Tween-80. Ten millilitres of this stock was added to NG agar medium (total $100 \mathrm{ml}$ ) after autoclaving and cooling to $60{ }^{\circ} \mathrm{C}$. For $\mathrm{PQT}$ and $\mathrm{CoQ}_{10}$ combined plates, paraquat was supplemented at a final concentration of $2 \mathrm{mM}$ in $\mathrm{NGM}$ agar containing $1 \mathrm{mg} / \mathrm{ml} \mathrm{CoQ_{10 }}$ $(1.158 \mathrm{mM})$. The lethality test was performed as described above.

\subsubsection{MitoQ assay}

MitoQ nematode treatment was performed as previously described $[37,38]$. One milligram of MitoQ was dissolved in $1 \mathrm{ml}$ of distilled water, and $250 \mu \mathrm{l}$ of this stock was added to $60{ }^{\circ} \mathrm{C}$ NG agar medium (total $50 \mathrm{ml}$ ) autoclaving and cooling to $60{ }^{\circ} \mathrm{C}$. We confirmed that $C$. elegans survival is not affected by $\mathrm{TPP}^{+}$and MitoQ at this concentration. For PQT and MitoQ $_{10}$ combined plates, PQT was supplemented at a final concentration of $4 \mathrm{mM}$ in NGM agar containing $5 \mu \mathrm{g} / \mathrm{ml}$ MitoQ $(7.3 \mu \mathrm{M})$. The lethality test was performed as described previously.

\subsubsection{Statistics}

All data are presented as the mean \pm SD. Group means were compared with either Student's t-test, one-way or two-way ANOVA, followed by Tukey's post hoc test. All $P$ values were two-tailed, and a $P$ value of less than 0.05 was considered statistically significant. All statistical analyses were analysed using SPSS software.

\section{Results}

\section{1. pmp-4 encodes the peroxisomal $A B C D 1$ orthologue, and pmp-}

4(ok396) loss of function mutants recapitulate the main hallmarks of X-ALD

Phylogenetic analysis identified pmp-4 as the orthologue and ancestor of mammalian peroxisomal transporters $A B C D 1$ and $A B C D 2$ in C. elegans [39]. PMP-4 shows $75 \%$ similarly to ABCD1 at the amino acid level, and $73 \%$ to ABCD2 (Supplementary Fig. S1A). In C. elegans, 5 orthologs of mammalian $\mathrm{ABCD}$ transporters exist: 1 for $\mathrm{ABCD} 1$ and $\mathrm{ABCD} 2$ (pmp-4), two for $\mathrm{ABCD} 3$ ( $p m p-1$ and pmp-2) and two for ABCD4 (pmp-3 and pmp-5) [39]. To identify the tissues in which pmp-4 is expressed, we used N2 worms harbouring the Ex001 [Ppmp-4::gfp] transcriptional reporter that expresses GFP under the control of the pmp-4 promoter and found that PMP-4 is mainly expressed in both the intestines and hypodermis (Supplementary Fig. S1B), which are the main tissues for fat storage in $C$. elegans [40]. Using an in silico mining tool for peroxisomal targeting sequences available at the peroxisome database (www.peroxisomedb.org) [41], we predicted the presence of the PEX19 binding site, a PTS signal for membrane proteins [42], between residues 140 to 151 of the PMP-4 coding sequence (Supplementary Fig. S1A), suggesting a peroxisomal localization for this transporter in the nematode. To validate the in silico prediction of the peroxisomal localization of PMP-4, we took advantage of another peroxisomal targeting signal, PTS type-1 (PTS1), which has been shown to target matrix proteins to peroxisomes in C. elegans [31]. Thus, using the strain hjIs37 [Pvha-6::rfp::PTS1], in which the peroxisomes are labelled with RFP in the intestine, we expressed PMP-4:GFP under the control of its own promoter and found the colocalization (yellow labelling) of both reporter proteins in the gut (Fig. 1A-D). Similarly, using the strain Ex095 [Pdpy-7::gfp::PTS1] that labels peroxisomes with GFP in the hypodermis, we expressed PMP-4:mCherry under the control of its own promoter and found colocalization in the hypodermis (yellow labelling)(Fig. 1E-H). These results confirm the presence of PMP-4 in peroxisomes in the two main peroxisomal-containing tissues in C. elegans, the gut and the hypodermis [43].

To establish a model of X-ALD in the nematode, we used a strain harbouring the pmp-4(ok396) allele, which contains an 867 bp deletion encompassing exons 6 to 10 (www.wormbase.org) (Supplementary Fig. S1C). pmp-4(ok396) worms did not show any obvious defects in growth or maturation.

We generated a polyclonal antibody using the last 21 amino acids of the C-terminal part of PMP-4 (Supplementary Fig. S1A) and performed Western blot (WB) experiments that detected a band above $75 \mathrm{kDa}$ in wild-type (WT, N2 strain) homogenates. This molecular weight is expected for a protein of 734 amino acids, while no protein was observed in pmp-4(ok396) extracts (Fig. 1I). As a positive control, we generated a transgenic strain expressing the PMP-4 protein fused to GFP at the Cterminus under the control of its own promoter in pmp-4(ok396) animals (pmp-4(ok396); Ex042 [Ppmp-4::pmp-4::gfp]) and used the homogenates for the WB (Fig. 1I). PMP-4 was not detected in pmp-4(ok396) animals by immunofluorescence (Fig. $1 \mathrm{~J}-\mathrm{K}$ ), demonstrating that pmp4(ok396) is a null allele. Furthermore, we observed that PMP-4 is well expressed from the first larval stage (L1) to adulthood, with higher expression from L3 onwards, whereas no expression was detected in embryos (Supplementary Fig. S1D).

The main biochemical hallmark of X-ALD is the accumulation of VLCFAs in complex lipids, especially in lysophosphatidylcholine (LPC) $[3,6,26]$, which is used as a robust diagnostic marker of X-ALD and, recently, in newborn screening $[3,26]$. We performed a tandem mass spectrometry analysis of LPC-VLCFAs from WT, pmp-4(ok396) and daf22(ok693) worms, the latter was used as a positive control for VLCFA accumulation (Fig. 1L). daf-22(ok693) mutants lack the last enzyme of the peroxisomal $\beta$-oxidation pathway called SCPx/thiolase and therefore accumulate VLCFAs [27]. Here, we observed that LPC-C20:0, LPC-C22:0, LPC-C24:0 and LPC-C26:0 were increased in both pmp-4(ok396) and daf22(ok693) worms (Fig. 1L), indicating a role for PMP-4 in importing VLCFAs into peroxisomes for degradation, similar to its mammalian orthologue. The difference in levels of VLCFA between pmp-4(ok396) and daf-22(ok693) mutants in C. elegans was not unexpected when compared with human data. Indeed, C26:0 was 1.7-fold higher accumulated in plasma from ACOX1 patients (the first rate-limiting enzyme of the peroxisomal $\beta$-oxidation) than in plasma from X-ALD patients [44]. In addition, de novo C26:0 synthesis was measured as the level of $\mathrm{D}_{3}-\mathrm{C} 26: 0$ produced from $\mathrm{D}_{3}-\mathrm{C} 22: 0$. Remarkably, $\mathrm{D}_{3}-\mathrm{C} 26: 0$ was increased in X-ALD fibroblasts and the effect exacerbated in the peroxisomal $\beta$-oxidation enzymes deficient fibroblasts (ACOX1-and HSD17B4-deficient fibroblasts) [45]. This difference of C26:0 levels between X-ALD patients and patients with peroxisomal $\beta$-oxidation deficiency is probably due to functional overlap between $\mathrm{ABCD} 1$ and $\mathrm{ABCD} 2$ peroxisomal transporters as previously reported $[16,39]$. Elegant experiments in yeast showed that the substrate specificities of $H s \mathrm{ABCD} 1, H s \mathrm{ABCD} 2$, and $H s \mathrm{ABCD} 3$ are overlapping even if they have distinctive preferences [46]. In C. elegans, there are 5 distinct $A B C D$ transporters which could also share redundant functions that could explain the difference of VLCFA levels between pmp4(ok396) and daf-22(ok693) mutants [39].

We next investigated whether impaired redox homeostasis is a phenotypic trait of pmp-4(ok396) nematodes. Redox immunoblotting with an antibody that recognizes dinitrophenol (DNP) modified protein carbonyls [20] showed that pmp-4(ok396) worms had significantly more oxidatively damaged proteins than did WT nematodes (Fig. 1M). This finding is correlated with higher levels of total reactive oxygen species (ROS) measured with $\mathrm{H}_{2}$ DCFDA probes [33] (Fig. $1 \mathrm{~N}-\mathrm{O}$ ), in agreement with evidence obtained in cellular, in vivo and ex vivo models of X-ALD [47-49]. Of note, the daf-22(ok693) strain did not display increased ROS production (Fig. 1N, P). We rescued the increased ROS production by using a construct containing PMP-4:mCherry driven by the pmp-4 promoter and injected into pmp-4(ok396) worms (pmp-4(ok396); Ex050 [Ppmp-4::pmp-4::mCherry]) (Fig. 1N-O, 1Q), in which $\mathrm{H}_{2}$ DCFDA staining 


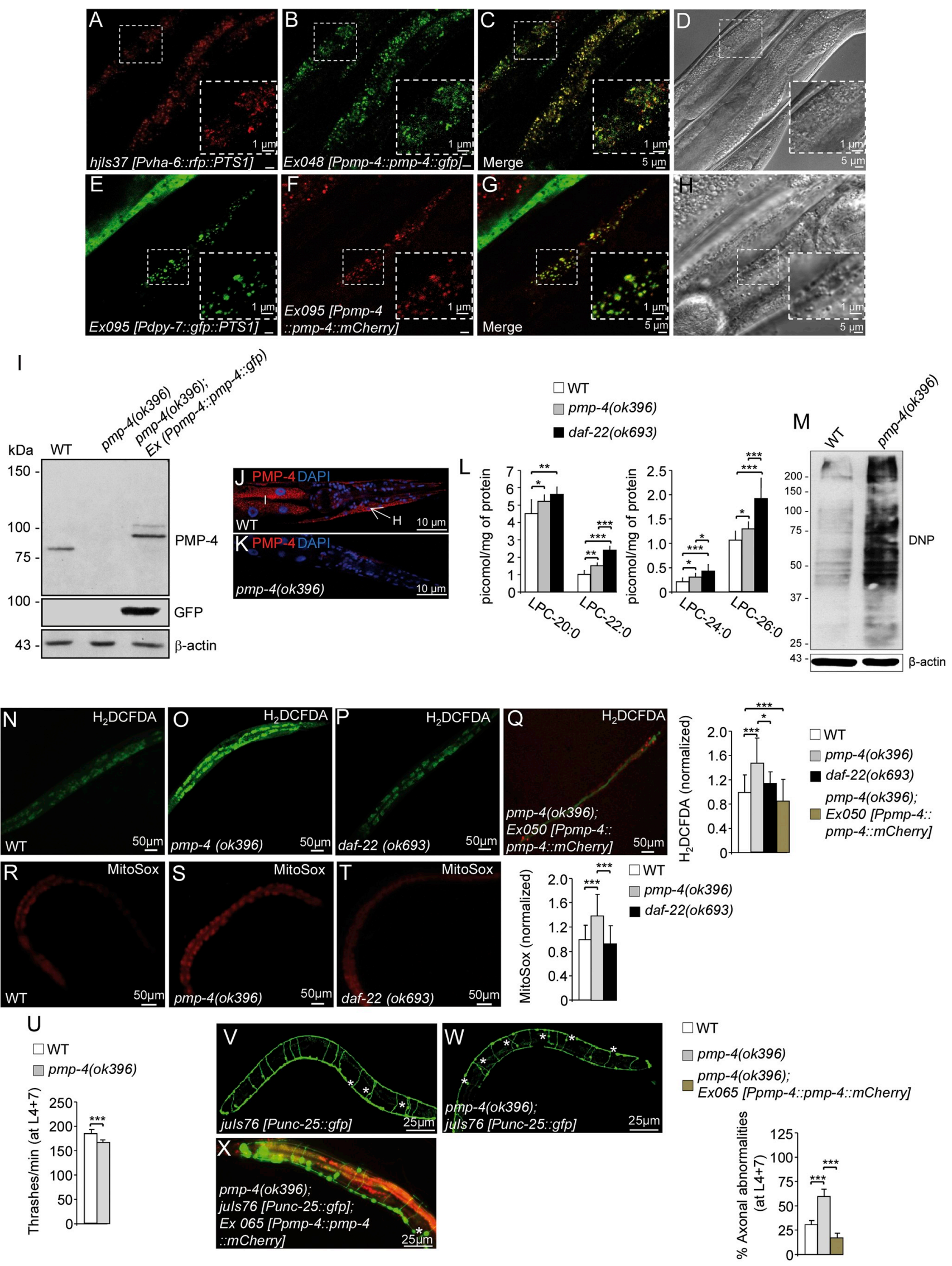

(caption on next page) 
Fig. 1. C. elegans pmp-4(ok396) mutants recapitulate the main hallmarks observed in X-ALD. (A-D) The construct containing PMP-4:GFP under the control of the pmp-4 promoter was injected into the strain labelling peroxisomes with RFP in the intestine (hjIs37 [Pvha-6p::rfp::PTS1]; Ex048 [Ppmp-4::pmp-4::gfp]). Images of (A) RFP:PTS1 and (B) PMP-4:GFP staining. (C) Merge of images A and B. In yellow, colocalization of RFP:PTS-1 with PMP-4:GFP. (D) DIC-Nomarsky pictures corresponding to the fluorescence images $(\mathrm{n}=30)$. $(\mathrm{E}-\mathrm{H})$ The constructs containing GFP:PTS1 under the control of a specific hypodermal promoter (Pdpy-7) and PMP-4:mCherry under the control of the pmp-4 promoter were coinjected in the N2 strain (Ex095 [Pdpy-7::gfp::PTS1 + Ppmp-4::pmp-4::mCherry]). Here, the peroxisomes are labelled with GFP in the hypodermis. Images of (E) GFP:PTS1 and (F) PMP-4:mCherry staining. (G) Merge of images E and F. In yellow, colocalization of GFP:PTS-1 with PMP-4:mCherry. (H) DIC-Nomarsky images corresponding to the fluorescence images $(\mathrm{n}=30)$. Scale $=5 \mu \mathrm{m}$ in each figure except for the expanded pictures in the squares, in which scale $=1 \mu \mathrm{m}$. The worms in the figures are oriented head to the left, dorsal face-up and only a portion of the central part of the body is depicted. (I) PMP-4 and PMP-4:GFP protein levels in wild-type (WT), pmp-4(ok396) and pmp-4(ok396) animals expressing PMP-4:GFP under the control of the pmp4 promoter (pmp-4(ok396); Ex042 [Ppmp-4::pmp-4::gfp]) at the L4 larval stage. $\beta$-actin was used as a loading control (bottom panel) (n $=4$ pools of worms by condition). (J-K) Immunofluorescence staining of formaldehyde-fixed (J) WT and (K) pmp-4(ok396) worms incubated with polyclonal anti-PMP-4 (red) and counterstained with DAPI (blue) at the L4 larval stage. The posterior part of the worm is depicted. I $=$ intestine and $\mathrm{H}=$ hypodermis. Scale $=10 \mu \mathrm{m}$. (L) Lysophosphatidylcholine fatty acid levels (LPC-20:0, LPC-22:0, LPC-24:0 and LPC-26:0) of L4 worm lysates of WT (n = 12), pmp-4(ok396)(n = 10) and daf22(ok693) animals $(\mathrm{n}=6$ ). The daf-22(ok693) mutant was used as a positive control. (M) Dinitrophenol (DNP) protein levels in WT and pmp-4(ok396) animals at the L4 larval stage. The quantification of these blots by densitometry was performed and normalized to $\beta$-actin ( $\mathrm{n}=4$ pools of worms by condition). Relative total ROS levels $\left(\mathrm{H}_{2}\right.$ DCFDA) were measured by quantifying the fluorescence emission of the $\mathrm{H}_{2}$ DCFDA probes in living animals in $(\mathrm{N}) \mathrm{WT}(\mathrm{n}=51),(\mathrm{O}) p m p-4(\mathrm{ok} 396)(\mathrm{n}=66)$, (P) daf-22(ok693) ( $\mathrm{n}=40$ ), and (Q) pmp-4(ok396) expressing PMP-4:mCherry under the control of the pmp-4 promoter (pmp-4(ok396); Ex050 [Ppmp-4::pmp4::mCherry]) $(\mathrm{n}=30) \mathrm{L} 4$ nematodes maintained in a liquid medium. Values are normalized to WT. Relative mitochondrial ROS levels in living animals in (R) WT ( $\mathrm{n}=66)$, (S) pmp-4(ok396) $(\mathrm{n}=88)$ and $(\mathrm{T})$ daf-22(ok693) $(\mathrm{n}=66) \mathrm{L} 4$ nematodes quantified with MitoSox probes. Values are normalized to WT worms. Scale $=50 \mu \mathrm{m}$. (U) The thrashing behaviour of WT and pmp-4(ok396) nematodes was analysed in liquid medium at L4 +7 days ( $\mathrm{n}=20$ animals/condition). Representative fluorescence images of GFP-labelled GABAergic neurons showing axonal abnormalities and quantitative analysis in living worms in (V) juIs76 [Punc25::gfp] ( $\mathrm{n}=20)$, (W) pmp-4(ok396); juIs76 [Punc-25::gfp] $(\mathrm{n}=20)$, and (X) pmp-4(ok396) where PMP-4:mCherry was expressed under the control of pmp-4 promoter (pmp-4(ok396); juls76 [Punc-25::gfp]; Ex065[Ppmp-4::pmp-4::mCherry])(n $=17$ ) at L4+7 days. Scale $=25 \mu$ m. Data represent the mean \pm standard deviation (SD). Statistical analysis was carried out with one-way ANOVA, followed by Tukey's post hoc test ( $\mathrm{P}<0.05$; **P $<0.01$; ***P $<0.001$ ).

was normalized. To investigate whether the origin of ROS in the absence of PMP-4 was mitochondrial, in a similar manner as in the fibroblasts of X-ALD patients [50], we incubated the pmp-4(ok396) worms with the MitoSOX probe, which specifically reacts with mitochondrial ROS [33]. The results indicated increased mitochondrial ROS in the mutant strain (Fig. 1R-S). In contrast, daf-22(ok693) did not produce higher mitochondrial ROS than WT (Fig. 1R, T), consistent with the data obtained when using a probe labelling total ROS (Fig. 1P). The absence of redox imbalance in daf-22(ok693) worms will be discussed later.

$\mathrm{X}$-ALD is characterized by the axonal degeneration of corticospinal tracts leading to spastic paraparesis in patients and locomotor disability in mouse models, as evidenced by rotarod, treadmill and bar-cross tests $[16,17,51]$. Here, we evaluated the motility of pmp-4(ok396) and WT strains by a thrashing assay, in which nematodes were placed in liquid, and the frequency of lateral swimming or thrashing movements was estimated [28]. Thrashing assays were performed blindly at L4 (Supplementary Fig. S2A) and L4 + 7 days (Fig. 1U, movies S1-S2), well after the worms reached adulthood. The pmp-4(ok396) mutants consistently thrashed at a significantly reduced rate when compared to the behaviour of WT at both ages tested in a comparable manner, thus indicating a defect in motility (Fig. 1U, Supplementary Fig. S2A, movies S1-S2). Finally, we examined axonal abnormalities by analysing the structural integrity of GABAergic D-type motor neurons and their processes in pmp-4(ok396) animals to evaluate whether locomotor phenotypes were associated with axonal degeneration, as noted in X-ALD mouse models and patients $[5,16,17]$. To address this question, we used the juIs76[Punc-25::gfp] strain with GFP-labelled GABAergic axons and cell bodies of the ventral cord [52]. We found significant axonal damage in pmp-4(ok396); juIs76 [Punc-25::gfp] worms compared to juIs76 [Punc-25::gfp] control animals, both before and after reaching adulthood, at L4 (Supplementary Figs. S2B-D) and L4 +7 days, respectively (Fig. 1V-W). Finally, we recovered axonal defects by injecting PMP-4 fused to mCherry into pmp-4(ok396) worms with GFP-labelled GABAergic cells (pmp-4(ok396); juIs76 [Punc-25::gfp]; Ex065 [Ppmp4::pmp-4::mCherry]) (Fig. 1V-X).

Supplementary video related to this article can be found at https:// doi.org/10.1016/j.freeradbiomed.2020.01.177

\subsection{The loss of pmp-4 induces lipid droplet accumulation in the nematode}

Lipid droplets (LDs) are dynamic organelles, such as peroxisomes, that emerge from the endoplasmic reticulum membrane and serve as a site for the storage of neutral lipids, such as triglycerides and cholesterol [53-55]. Cells can form LDs in response to stress conditions, such as inflammation or nutrient deprivation [56-58]. In C. elegans, the majority of lipids are stored in LDs and are mainly located in gut and hypodermal cells [59]. In other peroxisomal mutants, such as the $d h s$ 28(hj8) and daf-22(ok693) worms, which lack peroxisomal dehydrogenase and SCPx/thiolase, the third and fourth enzymes of the peroxisomal $\beta$-oxidation pathway, respectively, an increase in LDs is observed in the gut [31]. Thus, we hypothesized that LD formation could also be altered in pmp-4(ok396) mutants. We studied the presence of LDs using Sudan Black, a neutral lipid dye that stains LDs [60] and found that the number of lipid granules with a diameter $>5 \mu \mathrm{m}$ was significantly increased in pmp-4(ok396) worms compared to WT animals (Fig. 2A and C). We next applied a $24 \mathrm{~h}$ fasting period to the worms. This metabolic route was blunted in pmp-4(ok396) worms, which could not use their lipid reservoirs when most needed (Fig. 2A-D). Next, the increase in LDs visualized by Sudan black was no longer detected by using a construct containing PMP-4:mCherry driven by the pmp-4 promoter and injected into pmp-4(ok396) worms (pmp4(ok396); Ex050 [Ppmp-4::pmp-4::mCherry]) (Fig. 2A-E).

To complement these results with a specific marker of LDs, we chose adipose triglyceride lipase 1 (ATGL-1), which is a coating component of phospholipid monolayer delimiting LDs [61]. ATGL-1 fused to GFP protein localizes at the surface of large LDs in $d h s-28(h j 8)$ peroxisomal dehydrogenase mutant [31]. We crossed the strain hjIs67 [Patgl-1::atgl$1:: g f p]$ expressing ATGL-1:GFP [31] with the pmp-4(ok396) mutant and the results showed the improved visualization of the enlarged LDs when PMP-4 was absent (Fig. 2F-Q), similar to $d h s-28(h j 8)$ peroxisomal mutants [31], corroborating the inability of the pmp-4(ok396) worms to degrade LDs upon fasting (Fig. 2F-Q). In conclusion, pmp-4(ok396) mutants recapitulate the increased size of LDs observed in peroxisomal $\beta$-oxidation mutants, which is also refractory to fasting-induced lipolysis.

\subsection{Loss of PMP-4 increases vulnerability to mitochondrial ROS}

To directly test the sensitivity of pmp-4(ok396) worms to mitochondrial ROS, we incubated the animals with the pro-oxidant paraquat (PQT), a redox cycler that stimulates superoxide production in mitochondria at complex I [62]. Compared to WT animals, the pmp4(ok396) worms showed increased lethality under these conditions (Fig. 3A). Furthermore, we tested the impact of classical inhibitors of 
complexes II, III, and IV [63] on viability, revealing that pmp-4(ok396) mutants were more sensitive to inhibitors of complexes III and IV but not to inhibitors of complex II (Fig. 3B-E). We interpret these results as pmp-4(ok396) animals showing impaired defences against specific mitochondrial stressors.
3.4. The accumulation of lipid droplets in pmp-4(ok396) animals is reversed by mitochondrial antioxidants

To determine which of the phenotypes due to loss of PMP-4 were dependent on mitochondrial redox imbalance, we used two antioxidants targeting mitochondria, Coenzyme $\mathrm{Q}_{10}(\mathrm{CoQ})$ and MitoQ. MitoQ is a

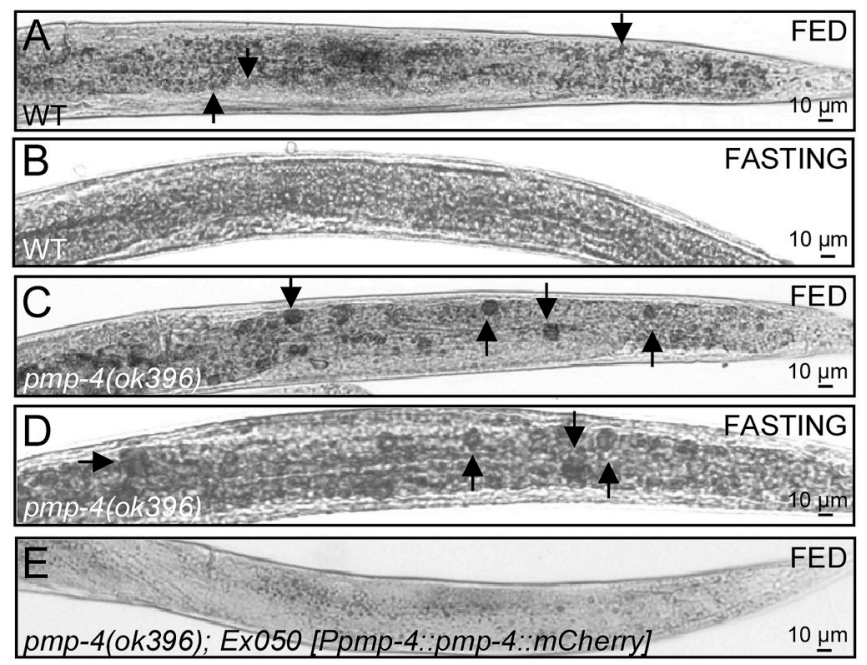

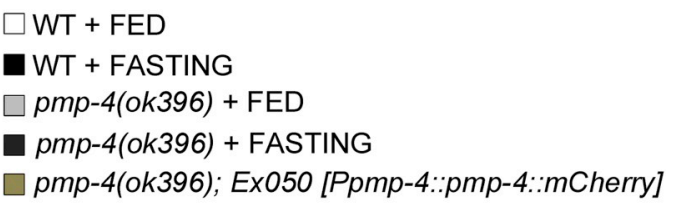

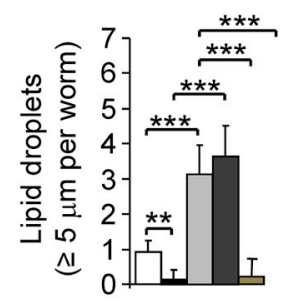

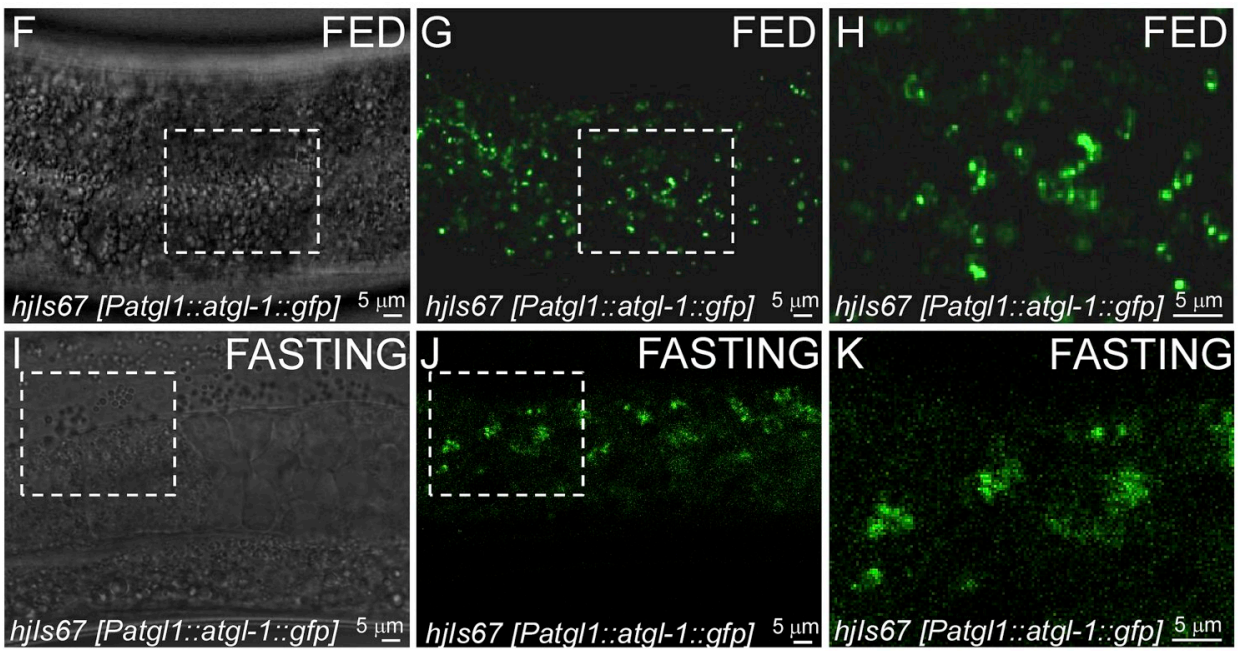
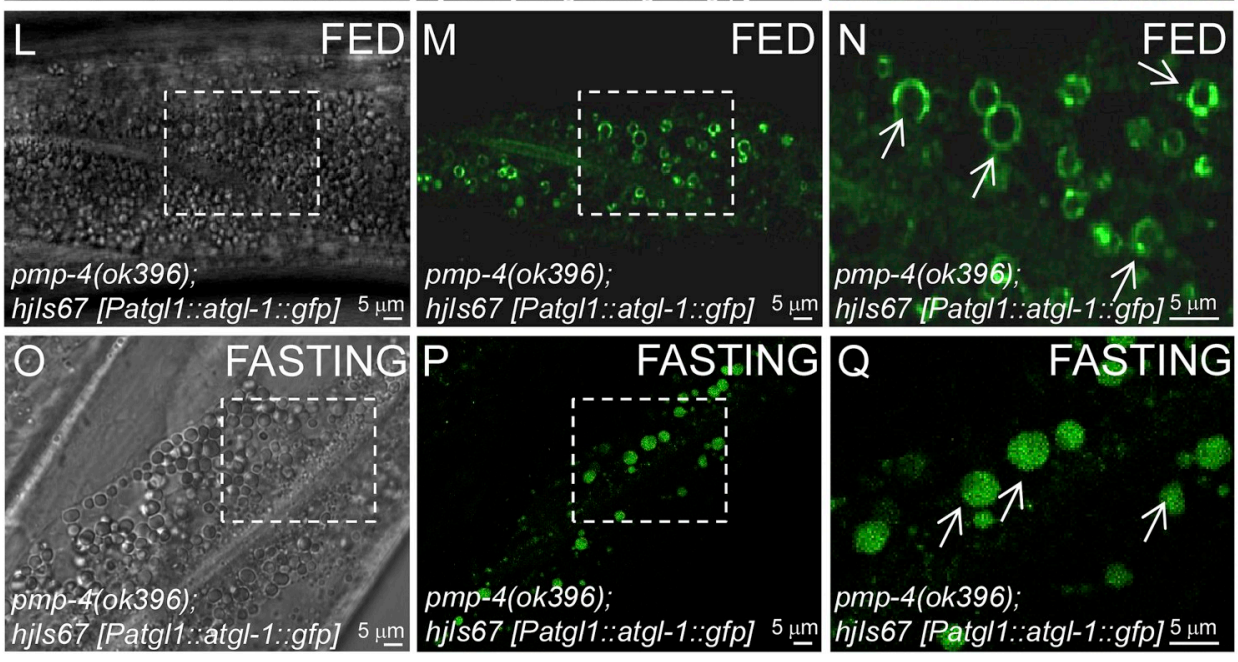


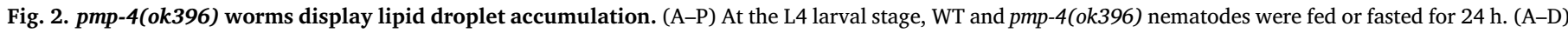

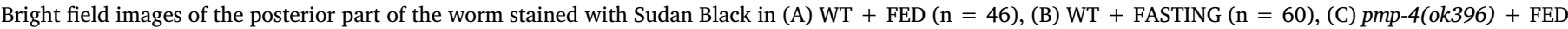

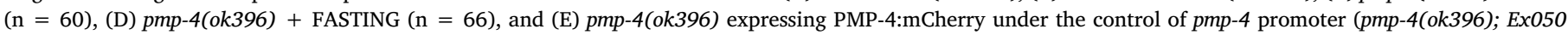

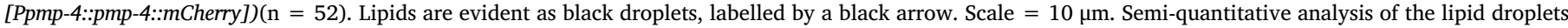

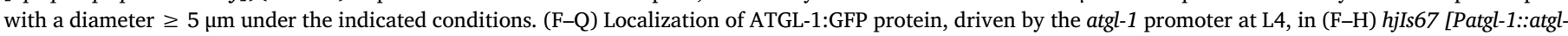

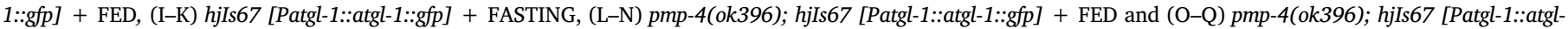

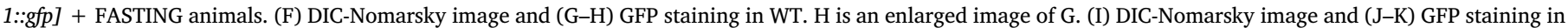

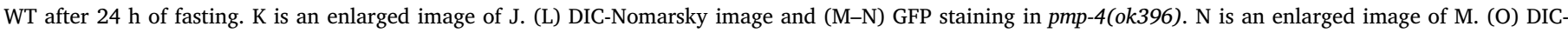

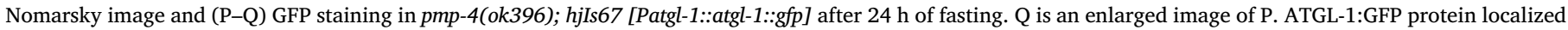

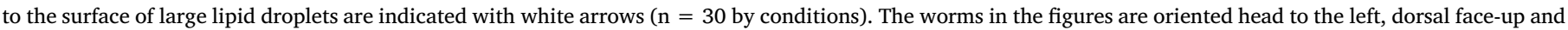

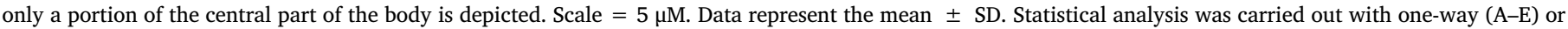
two-way ANOVA (F-Q), followed by Tukey's post hoc test $\left({ }^{*} \mathrm{P}<0.05 ;{ }^{* *} \mathrm{P}<0.01 ; * * * \mathrm{P}<0.001\right)$.

A

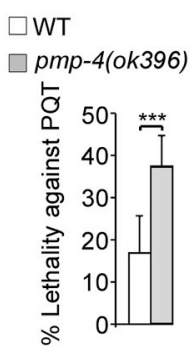

B

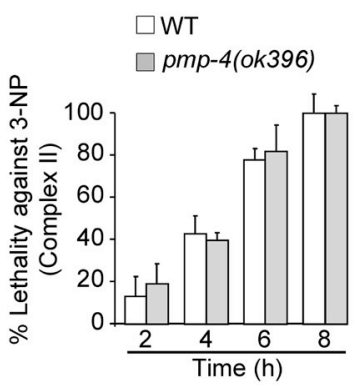

C

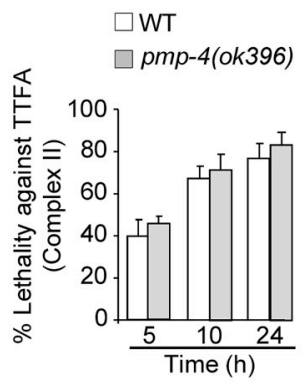

D

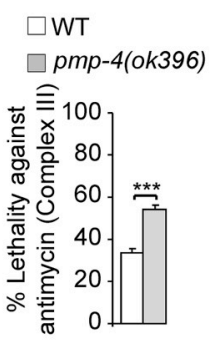

$E$

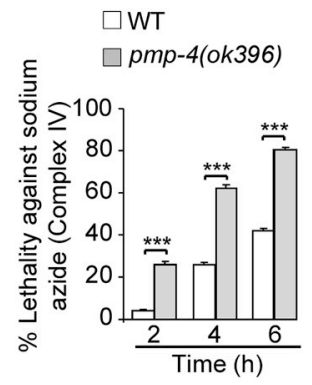

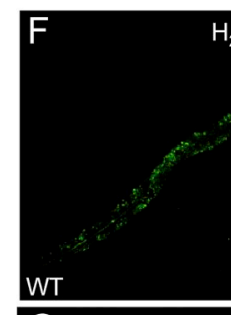
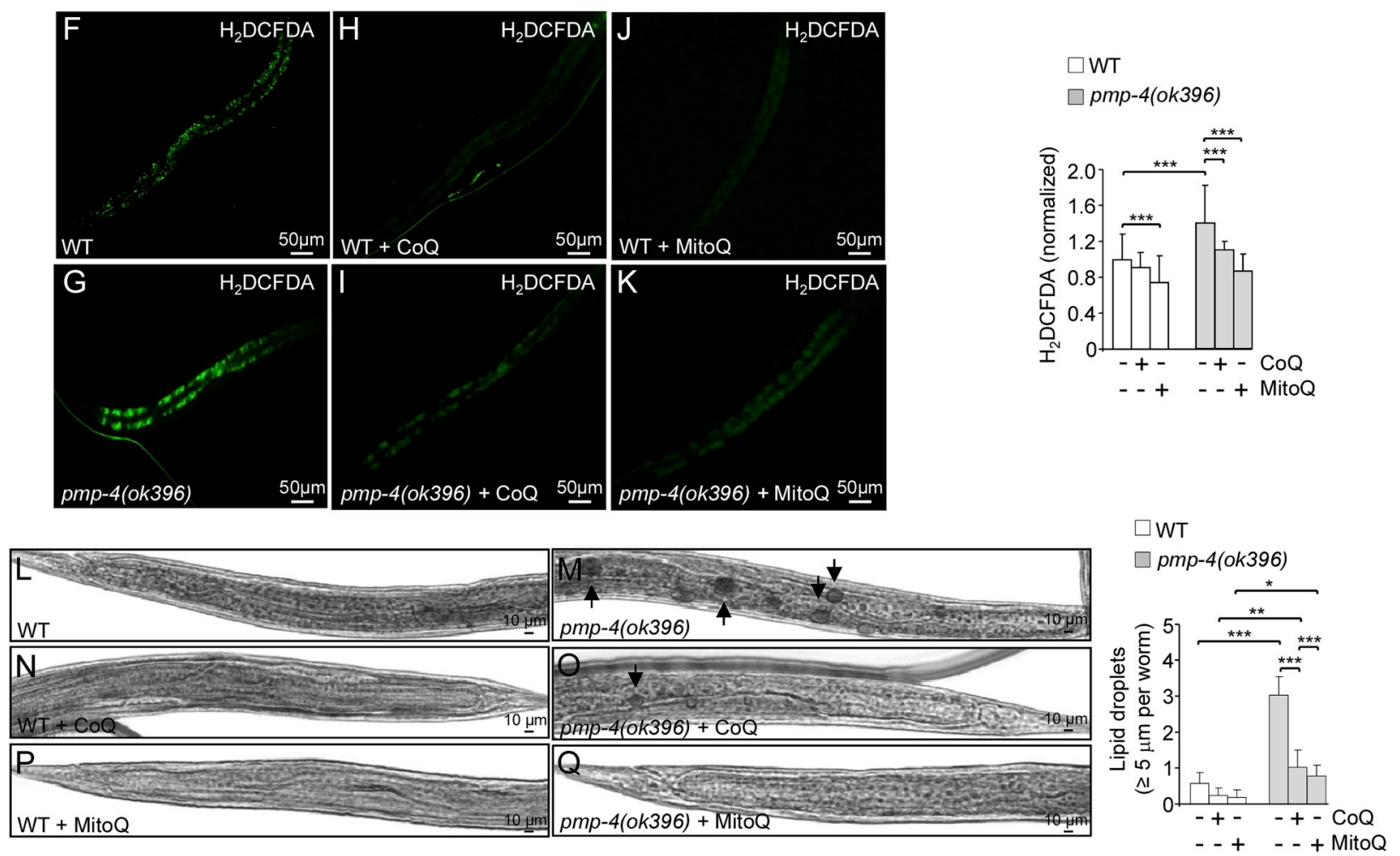

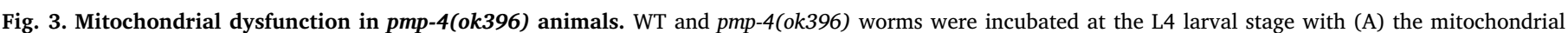

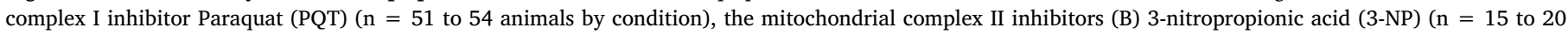

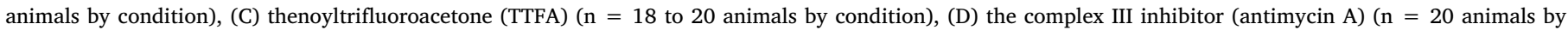

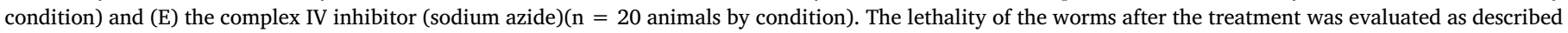

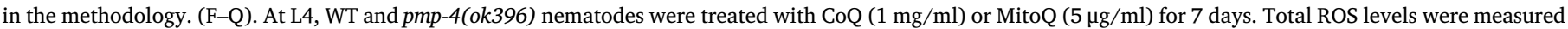

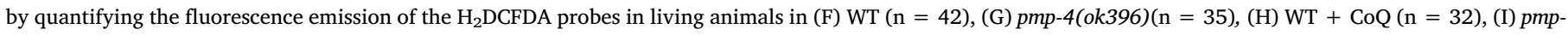

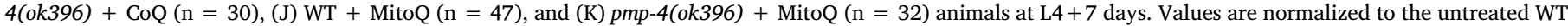

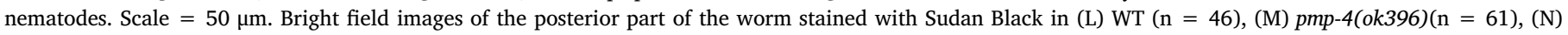

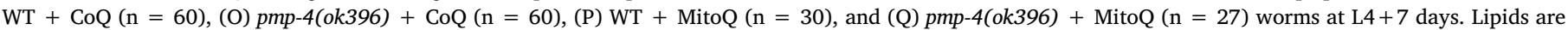

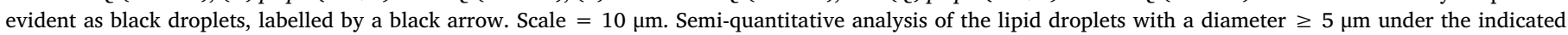

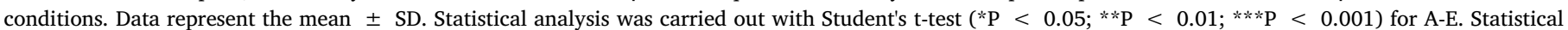
analysis was carried out with two-way ANOVA, followed by Tukey's post hoc test (*P $<0.05 ; * * \mathrm{P}<0.01$; ***P $<0.001$ ) for F-Q. 
modified form of ubiquinol attached to a lipophilic cation that enables it to cross cell membranes and accumulate preferentially on the matrixfacing the surface of the mitochondrial inner membrane, where it is optimally positioned to decrease most types of mitochondrial ROS [37]. We showed that ROS levels were restored by treatment with both compounds, although much lower doses of MitoQ were necessary (200-fold less compared to $\mathrm{CoQ}$ ) (Fig. 3F-K), underscoring the mitochondrial origin of the redox imbalance in the absence of PMP-4. Next, we incubated pmp-4(ok396) with CoQ and MitoQ to investigate whether the observed increase in LDs involved a mitochondrial redox-dependent mechanism. Remarkably, the enlargement of LDs could be normalized upon treatment with these two mitochondrial antioxidants (Fig. 3L-Q).

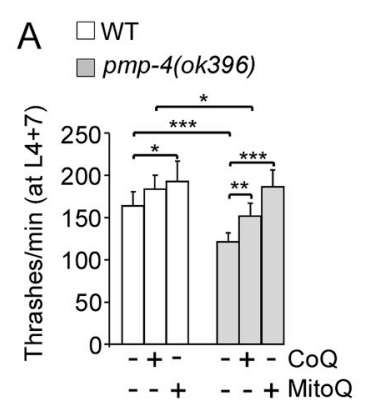

$\square$ juls76 [Punc-25::gfp]

$\square$ pmp-4(ok396);

$\square$ juls76 [Punc-25::gfp]

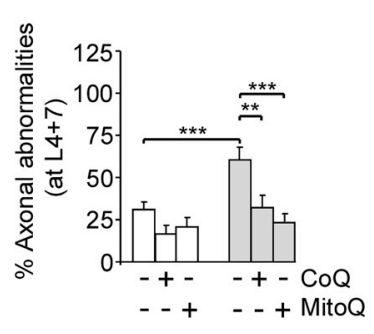

$\mathrm{H} \quad \square \mathrm{WT}$

$\square p m p-4(0 k 396)$

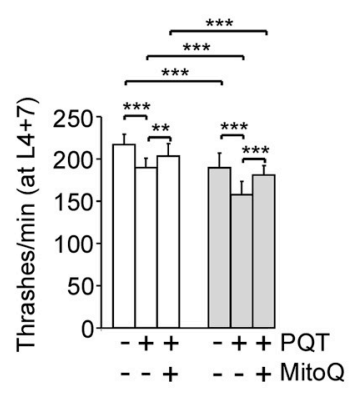

$\square$ juls76 [Punc-25::gfp]

$\square$ pmp-4(ok396);

juls76 [Punc-25::gfp]

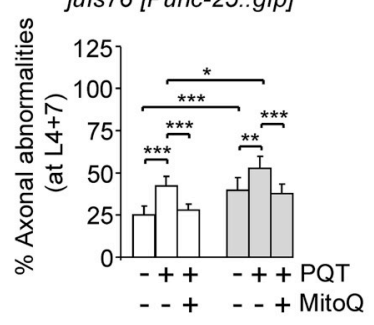

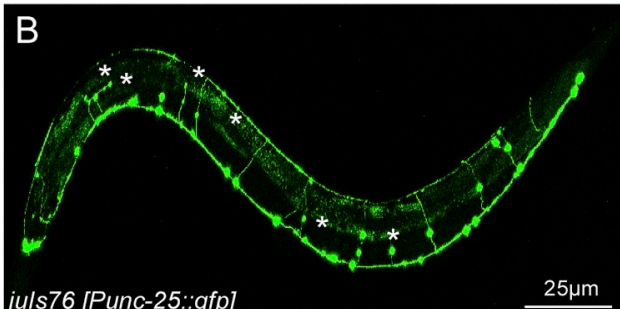
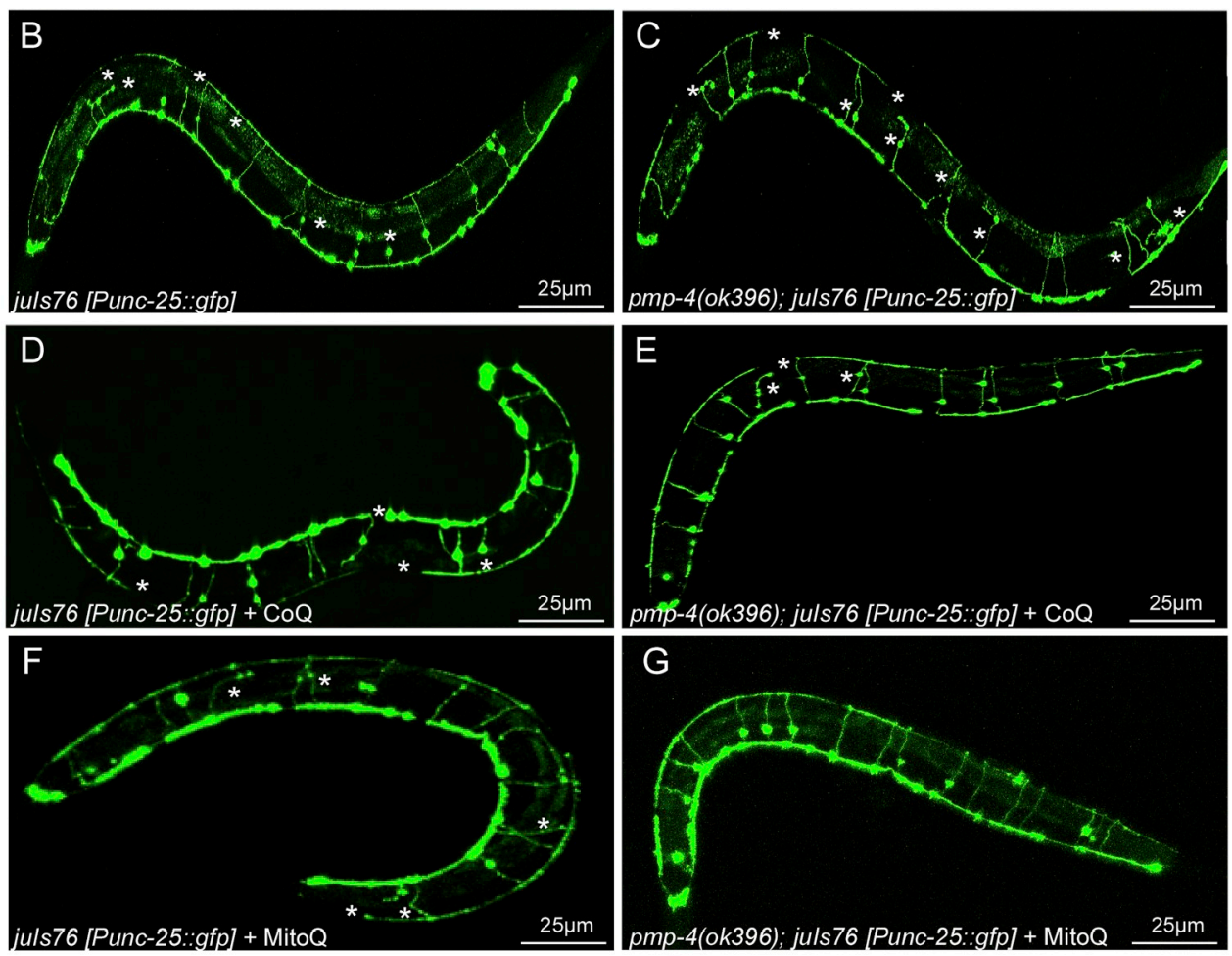

G

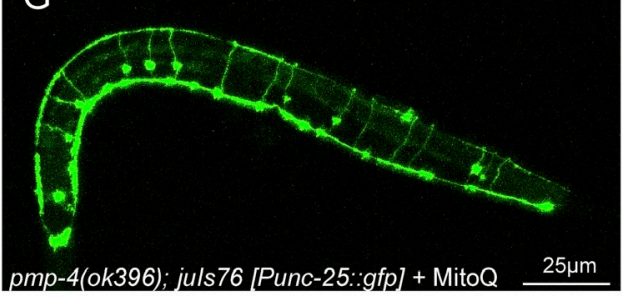

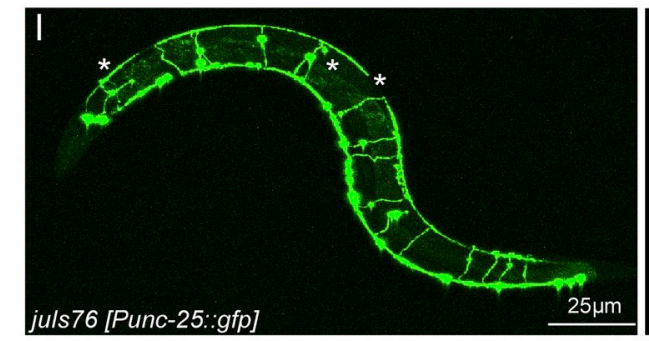
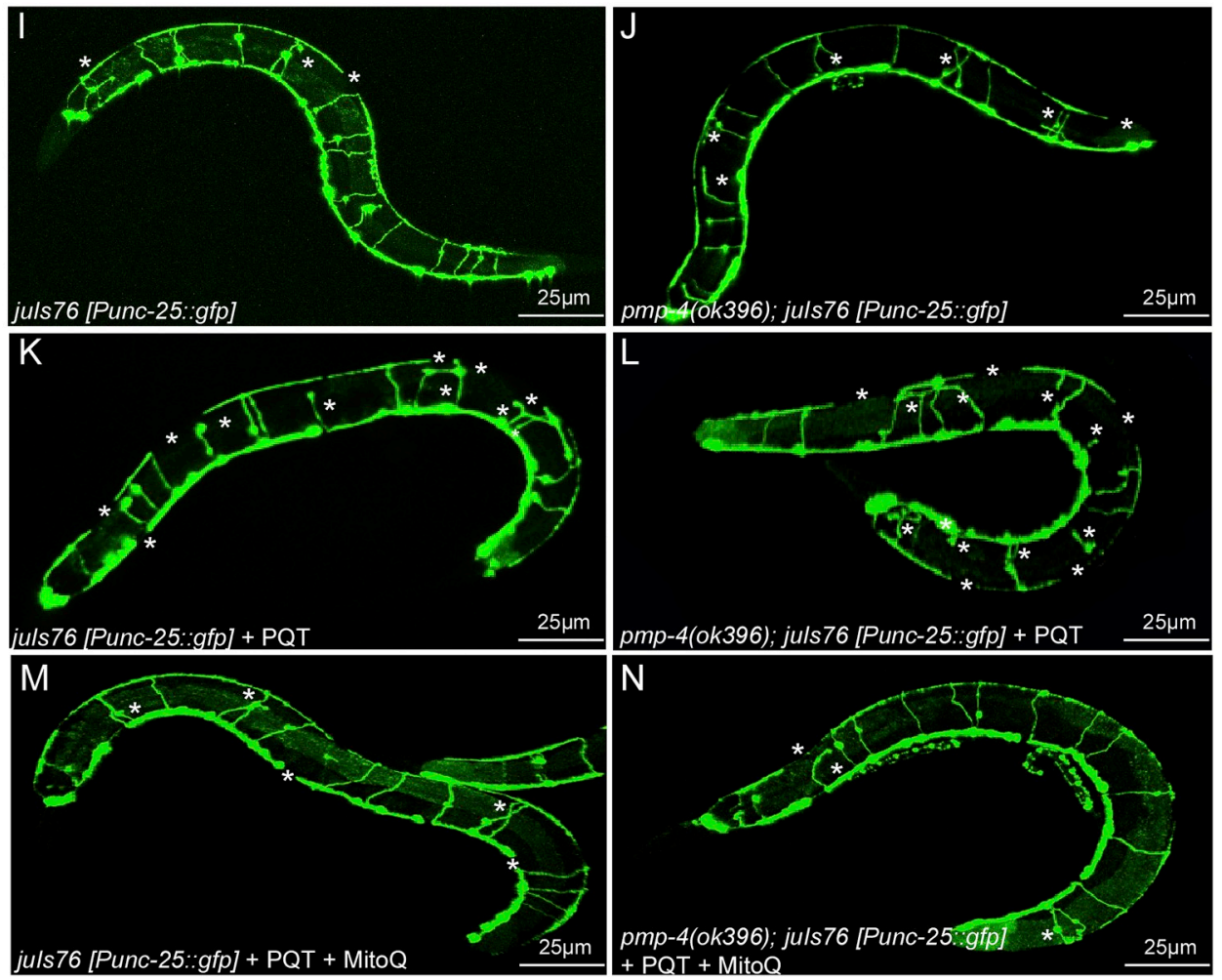

(caption on next page) 
Fig. 4. Mitochondrial-targeted antioxidant rescue axonal abnormalities and locomotor dysfunction at old stages of development. (A-G) At the L4 larval stages, WT and pmp-4(ok396) nematodes were treated with CoQ ( $1 \mathrm{mg} / \mathrm{ml})$ or MitoQ (5 $\mu \mathrm{g} / \mathrm{ml})$ for 7 days. (A) The thrashing behaviour was carried out in WT and pmp-4(ok396) nematodes upon CoQ or MitoQ at $\mathrm{L} 4+7$ days ( $\mathrm{n}=15$ to 27 animals by condition). Representative fluorescence images showing axonal abnormalities and quantitative analysis at the indicated genotypes upon CoQ or MitoQ at L4+7 days in (B) juls76 [Punc-25::gfp] (n = 20), (C) pmp-4(ok396); juls76 [Punc-25::gfp] ( $\mathrm{n}=20)$, (D) juIs76 [Punc-25::gfp] + CoQ (n = 18), (E) pmp-4(ok396); juIs76 [Punc-25::gfp] + CoQ (n = 15), (F) juIs76 [Punc-25::gfp] + MitoQ (n = 26) and (G) pmp-4(ok396; juIs76 [Punc-25::gfp]) + MitoQ ( $\mathrm{n}=27$ ). White asterisks indicate axonal abnormalities. All worms are oriented with the anterior end left and ventral side down. Scale $=25 \mu \mathrm{m}$. (H-N) At the L4 larval stage, juIs76 [Punc-25::gfp] and pmp-4(ok396); juIs76 [Punc-25::gfp] nematodes were treated with paraquat (PQT) $(0.2 \mathrm{mM})$ and/or MitoQ $(5 \mu \mathrm{g} / \mathrm{ml})$ for 7 days. (H) The thrashing behaviour was analysed at stage L4 + 7 days in WT and pmp-4(ok396) nematodes upon PQT and/or MitoQ ( $\mathrm{n}=20$ to 31 animals by condition). Representative fluorescence images showing axonal abnormalities and quantitative analysis at stage L4 + 7 days in (I) juIs76 [Punc-25::gfp]) (n = 29), (J) pmp-4(ok396); juIs76 [Punc-25::gfp] ( $=31)$, (K) juls76 [Punc-25::gfp] + PQT(n = 25), (L) pmp-4(ok396); juIs76 [Punc25::gfp] + PQT (n = 20), (M) juIs76 [Punc-25::gfp] + PQT + MitoQ (n = 21), (N) pmp-4(ok396; juIs76 [Punc-25::gfp] + PQT + MitoQ (n = 28). Scale = 25 $\mu$ m. All worms are oriented with the anterior end left and ventral side down. Data represent the mean \pm standard deviation (SD). Statistical analysis was carried out with two-way ANOVA, followed by Tukey's post hoc test (*P $<0.05$; **P $<0.01$; *** $\mathrm{P}<0.001)$ for A-N.

\subsection{Axonal degeneration in pmp-4(ok396) mutants is caused by} mitochondrial ROS

Next, we examined the effect of CoQ and MitoQ on axonal defects and locomotion disabilities in the pmp-4(ok396) worms. Thrashing abnormalities were restored by CoQ, but more efficiently by the mitochondrial-targeted MitoQ, at L4 (Supplementary Fig. S2E) and L4+7 days (Fig. 4A). This effect was correlated with an improvement of axonal damage with CoQ and MitoQ at L4+7 days (Fig. 4B-G).

We further investigated the role of mitochondrial ROS production in the maintenance of locomotion and axonal health. To address a causeeffect relationship, we evaluated the thrashing behaviour and axonal morphology in N2 and pmp-4(ok396) worms exposed to PQT at a $200 \mu \mathrm{M}$ sublethal dose at $\mathrm{L} 4+7$ days (Fig. 4H-N). PQT treatment reduced the locomotion fitness and increased axonal damage in both N2 and pmp-4(ok396) worms, with an exacerbated effect in pmp-4(ok396) worms (Fig. 4H-L). Importantly, MitoQ normalized these abnormalities (Fig. 4H-N).

To provide orthogonal evidence for the axonal defects caused by damage to the mitochondrial oxidative phosphorylation (OXPHOS) system, we performed RNAi knockdown of the mitochondrial gene nuo1, encoding the NDUFB4/B15 subunit of complex I required for OXPHOS, in WT worms [64]. We found that nuo-1 RNAi worms recapitulated the axonal defects present in the GABAergic neurons of pmp-4(ok396) and PQT-treated worms at L4 +7 days (Supplementary Figs. S2F-G). Altogether, these results indicated that mitochondrial ROS generated by the loss of PMP-4 are detrimental for axonal integrity, causing defects in locomotion.

3.6. PMP-4 expression in the hypodermis rescues locomotor disability and axonal degeneration through cell non-autonomous mechanisms

We then attempted to restore PMP-4 in the tissues where its endogenous expression was higher by expressing PMP-4:mCherry in the hypodermis (Ex177 [Pdpy-7::pmp-4::mCherry]) or in the intestine (Ex151 [Pges-1::pmp-4::mCherry]), using the strain previously used for characterizing the axonal defects, pmp-4(ok396); juIs76 [Punc-25::gfp], as background (Fig. 5A-E). We also expressed PMP-4:mCherry in GFPlabelled GABAergic cells (pmp-4(ok396); juIs76 [Punc-25::gfp]; Ex191 [Punc-25::pmp-4::mCherry]), as additional confirmation (Fig. 5A and F). Locomotion defects were only rescued when PMP-4:mCherry was expressed in the hypodermis but not in the intestine or in GABAergic neurons (Fig. 5A, movies S3-S7). Axonal damage was also rescued by restoring the expression of PMP-4:mCherry in hypodermal cells (Fig. 5B-D) but not in intestinal cells (Fig. 5B-C, 5E) or in GABAergic cells (Fig. 5B-C, 5F). Collectively, these results provide evidence that restoring the function of PMP-4 in the hypodermis is necessary and sufficient to maintain axonal integrity and locomotion in a cell nonautonomous manner.

Supplementary video related to this article can be found at https:// doi.org/10.1016/j.freeradbiomed.2020.01.177

\section{Discussion}

This work provides a novel animal model for a dreadful neurometabolic disorder, which, beyond the practicalities of cost-beneficial in vivo drug screening, delivers fundamental insights for an improved understanding of its molecular pathogenesis. pmp-4 deficient worms recapitulate the main hallmarks observed in human AMN patients and $A b c d 1^{-}$mice, i.e., the accumulation of VLCFAs, the mitochondrial redox imbalance, and most importantly, the axonal degeneration and associated locomotor dysfunction $[5,18,65]$, indicating that the mechanisms leading to disease upon PMP-4 dysfunction are evolutionarily conserved. We have previously shown that oxidative damage is a direct consequence of fatty acid excess and appears well in advance of the onset of symptoms $[47,50]$ and that the combination of antioxidants $\mathrm{N}$ acetylcysteine, lipoic acid and vitamin E could halt axonal degeneration in the mouse model [49]. Our working hypothesis is based on the interference of intracellular excess VLCFAs or membrane lipidic components containing lateral chains of VLCFAs with OXPHOS assembly or interaction, which leads to the generation of excess ROS and diminish ATP at this site [66]. The study of PMP-4 function in C. elegans expands these findings and provides precise evidence of enhanced mitochondrial vulnerability, since inhibitors of complexes I, III and IV dramatically increase pmp-4(ok396) lethality. We also show a direct causative role for mitochondrial redox dysfunction in axonal demise, since the selective mitochondrial antioxidant MitoQ prevented axonal degeneration. Altogether these results strengthen the rationale for using antioxidants targeting mitochondria to modify the progression of this peroxisomal disease, such as MitoQ, which has been used safely in phase II trials for Parkinson's [67] and has shown clinical efficacy in vascular function in healthy elderly individuals [68].

It is worth noting that we did not observe redox imbalance in the peroxisomal thiolase, beta-oxidation defective daf-22(ok693) mutant. This is also the case when the first limiting enzyme of peroxisomal betaoxidation, the acetyl-CoA oxidase, is silenced in HepG2 cells [69]. A plausible explanation may be that, in these mutants, VLCFA enter peroxisomes and presumably remain inside the organelles, thus preserving mitochondria membrane fluidity.

Regarding the biochemical defect presented by the pmp-4(ok396) mutants, we observed a modest 1.25-fold increase in LPC-C26:0 levels compared to WT. These differences are lower than the three to six fold increases found in plasma and tissues of patients and mouse models $[26,70]$, but consistent with the C26:0 levels detected in the zebrafish model where abcd1 gene is deleted [71]. The lower levels of C26:0 in both animal models compared to humans may owe to the fact that C26:0 have been quantified in whole fish or worms. In contrast, the excess of C26:0 levels is variable in the mouse depending of the tissues, ranging from 1.5-fold in the liver to 6 -fold in the sciatic nerve $[16,72]$.

The finding of expanded LD compartment in pmp-4(ok396) mutants is intriguing. Similar increases in LD in glial cells in drosophila has been described as a result of oxidative stress-either originated under hypoxic conditions or due to excess free radicals [73]. The authors attributed to the increased $\mathrm{LD}$ a protective effect through the sequestration of 
vulnerable membrane fatty acids away from free radicals, thus avoiding a devil circle of lipid peroxidation [73]. On the other hand, an emerging role for LDs in the pathogenesis of neurodegenerative disease is evidenced by the identification of several key regulators as causative genes of corticospinal axonal demise, such as Spastin, Reep1, or Atlastin-1 $[74,75]$. Reports on the increase or enlargement of LDs in peroxisomal

A
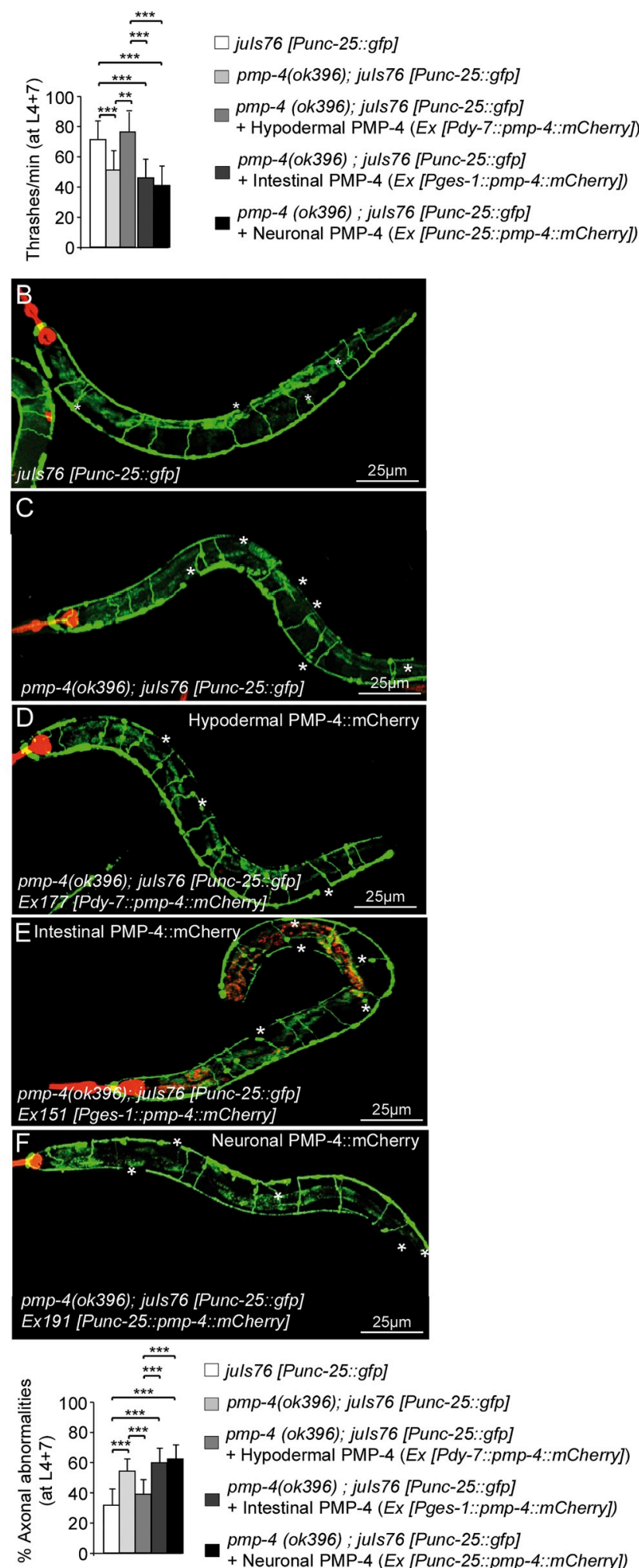

Fig. 5. Specific PMP-4 expression in the hypodermis is essential to maintain axonal integrity and locomotion. (A) Locomotion behaviour expressed as thrashes per minute at L4 +7 days in juIs76 [Punc-25::gfp], pmp-4(ok396); juIs76 [Punc-25::gfp] and pmp-4(ok396; juIs76 [Punc-25::gfp]) animals where PMP-4:mCherry is expressed in hypodermis (pmp-4(ok396); juIs76 [Punc25::gfp]; Ex177 [Pdpy-7::pmp-4::mCherry]), in intestine (pmp-4(ok396); juIs76 [Punc-25::gfp]; Ex151 [Pges-1::pmp-4::mCherry]) or in neurons (pmp-4(ok396); juIs76 [Punc-25::gfp]; Ex191 [Punc-25::pmp-4::mCherry]) ( $\mathrm{n}=19$ to 23 animals by condition). Representative confocal pictures showing axonal damage and quantitative analysis in (B) juIs76 [Punc-25::gfp] $(\mathrm{n}=22)$, (C) pmp-4(ok396); juIs76 [Punc-25::gfp] animals $(\mathrm{n}=19)$, pmp-4(ok396) animals where PMP4:mCherry is expressed in (D) hypodermis (pmp-4(ok396); juIs76 [Punc-25::gfp]; Ex177 [Pdpy-7::pmp-4::mCherry]) ( $=23)$, (E) intestine (pmp-4(ok396); juIs76 [Punc-25::gfp]; Ex151 [Pges-1::pmp-4::mCherry]) $(\mathrm{n}=22)$ or $(\mathrm{F})$ neurons (pmp4(ok396); juIs76 [Punc-25::gfp]; Ex191 [Punc-25::pmp-4::mCherry]) ( $=19$ ). The red colour in all pictures corresponds to the strong expression in the pharynx of the co-injection marker Pmyo-2::mCherry. White asterisks label the axonal abnormalities. Worms are oriented head to the left and dorsal face-up. Scale $=25 \mu \mathrm{m}$. Data represent the mean \pm standard deviation (SD). Statistical analysis was carried out with two-way ANOVA, followed by Tukey's post hoc test $\left({ }^{*} \mathrm{P}<0.05 ; * * \mathrm{P}<0.01 ;{ }^{* * *} \mathrm{P}<0.001\right)$.

disorders are scarce but include a mouse mutant of the peroxisomal biogenesis factor PEX5, causing Zellweger syndrome [76]. C. elegans responds to starvation by activating a number of genes involved in lipid metabolism, including the mitochondrial and peroxisomal $\beta$-oxidation genes, as these organelles cooperate in LD degradation [39,77]. During fasting, when the main fuel arrives from fatty acid stores, the loss of PMP-4 may impede their degradation in peroxisomes. We hypothesize that healthy mitochondria may compensate for the loss of peroxisomal function for degrading LD-derived fatty acids. This compensation may explain why treatment with antioxidants, which may exert protective effects on mitochondrial function and boost $\beta$-oxidation [78], normalizes the expanded LD compartment increasing the degradation of the fatty acids contained inside. This relationship appears reciprocal, as the production of mitochondrial ROS in neurons activates pathways inducing LD formation in glial cells, in a cell non-autonomous manner, in Drosophila and mouse models of OXPHOS impairment [79]. Moreover, increased LDs have been shown to be detrimental under conditions of marked mitochondrial oxidative stress, causing the enhancement of lipid peroxidation and neurodegeneration in Drosophila and mouse models overexpressing the fatty acid transporter FATP [80]. Based on the strong body of evidence implicating mitochondrial dysfunction and particularly the OXPHOS system in the pathogenetic cascade in X-ALD [14,48,50,51], we posit that the negative impacts of mitochondrial redox imbalance may be exacerbated by LD accumulation, leading to axonal degeneration. Elucidation of the precise mechanism orchestrating the regulation of LD formation by redox signalling may bear therapeutic potential and impact for neurodegenerative conditions in which mitochondria and LD dyshomeostasis converge.

The ectopic expression of PMP-4:mCherry in the hypodermis of pmp4(ok396) nematodes was sufficient to halt axonal degeneration, while no effect was observed when PMP-4:mCherry was expressed in the intestine or in neurons. This evidence indicates a fundamental metabolic role for the peroxisome compartment in the hypodermis that is necessary to maintain axonal integrity via a cell non-autonomous mechanism, which is supported by previous data in mice. The model shows that oligodendrocyte-specific ablation of functional peroxisomes through inactivation of the Pex 5 gene, is sufficient to cause severe axonal degeneration [81].

Indeed, C. elegans hypodermal cells participate in guiding neuronal migration and share characteristics with vertebrate glial cells [82], enveloping neuronal cell bodies and their processes and supporting neuronal function and architecture [83]. During embryogenesis, the hypodermis is implicated in neuronal migration [84] and later in development, axons grow completely embedded into the hypodermis $[83,84]$. Thus, it is likely that the strict physical contact may facilitate 
the passage of signalling or nurturing metabolites, such as essential lipids, from peroxisomes in the hypodermis towards axons. For instance, the neuroprotective DHA (C22:6 63 ) is synthetized in peroxisomes by the $\beta$-oxidation of $\mathrm{C} 24: 6 \omega 3$, which is imported into peroxisomes by $\mathrm{ABCD} 1$ and ABCD2 transporters, the mouse orthologues of PMP-4 [39]. A model of laser-induced axotomy provides evidence of a cell non-autonomous mechanism implicating the hypodermis in the maintenance of axonal integrity [85]. Thus, we posit that hypodermis may play a key supporting role in the protection and maintenance of neurons and axons in nematodes, similar to myelin in mammals, underscoring the importance of this tissue in models of axonal or neuronal degeneration in C. elegans.

\section{Declaration of competing interest}

The authors declare no conflict of interest.

\section{Acknowledgements}

We thank CERCA Program/Generalitat de Catalunya for institutional support. Most of the strains were supplied by the Caenorhabditis Genetic Center (CGC). Plasmids HYM772 was kindly provided by Dr. Ho Yi Mak (Stowers Institute for Medical Research, Kansas City, MO, USA). We especially thank Dr. Marta Artal, Centro Andaluz de Biología del Desarrollo, Sevilla, Spain, for critical reading the manuscript. This work was supported by grants from the Autonomous Government of Catalonia [2017SGR1206] to A.P., The Spanish Ministry of Science and Competitivity grants (PC0009/003 and PI1100968 to E. D). This study has been funded by Instituto de Salud Carlos III through the grants [Miguel Servet program CPII16/00016] to S.F. (Co-funded by European Social Fund. ESF investing in your future), and the Center for Biomedical Research on Rare Diseases (CIBERER) to A.P and M.R. S.G. was a fellow of the Autonomous Government of Catalonia [2014FI_B2 00028]. A.C. and J.P. were fellows of IDIBELL.

\section{Appendix A. Supplementary data}

Supplementary data to this article can be found online at https:// doi.org/10.1016/j.freeradbiomed.2020.01.177.

\section{References}

[1] P.P. Van Veldhoven, M. Baes, Peroxisome deficient invertebrate and vertebrate animal models, Front. Physiol. 4 (2013) 335.

[2] H.R. Waterham, S. Ferdinandusse, R.J. Wanders, Human disorders of peroxisome metabolism and biogenesis, Biochim. Biophys. Acta 1863 (5) (2016) 922-933.

[3] A.B. Moser, A. Fatemi, Newborn screening and emerging therapies for X-linked adrenoleukodystrophy, JAMA Neurol. 75 (10) (2018) 1175-1176 e.

[4] C.W. van Roermund, W.F. Visser, L. Ijlst, A. van Cruchten, M. Boek, W. Kulik, H.R. Waterham, R.J. Wanders, The human peroxisomal ABC half transporter ALDP functions as a homodimer and accepts acyl-CoA esters, Faseb. J. 22 (12) (2008) 4201-4208.

[5] I. Ferrer, P. Aubourg, A. Pujol, General aspects and neuropathology of X-linked adrenoleukodystrophy, Brain Pathol. 20 (4) (2010) 817-830.

[6] H. Moser, K.D. Smith, P.A. Watkins, J. Powers, A.B. Moser, X-linked adrenoleukodystrophy, in: C. Scriver (Ed.), The Metabolic and Molecular Bases of Inherited Disease, McGraw-Hill, New-York, 2001, pp. 3257-3301.

[7] P. Aubourg, S. Blanche, I. Jambaque, F. Rocchiccioli, G. Kalifa, C. Naud-Saudreau, M.O. Rolland, M. Debre, J.L. Chaussain, C. Griscelli, et al., Reversal of early neurologic and neuroradiologic manifestations of X-linked adrenoleukodystrophy by bone marrow transplantation, N. Engl. J. Med. 322 (26) (1990) 1860-1866.

[8] J.S. Kuhl, F. Suarez, G.T. Gillett, P.G. Hemmati, J.A. Snowden, M. Stadler, G.L. Vuong, P. Aubourg, W. Kohler, R. Arnold, Long-term outcomes of allogeneic haematopoietic stem cell transplantation for adult cerebral X-linked adrenoleukodystrophy, Brain 140 (4) (2017) 953-966.

[9] N. Cartier, S. Hacein-Bey-Abina, C.C. Bartholomae, G. Veres, M. Schmidt, I. Kutschera, M. Vidaud, U. Abel, L. Dal-Cortivo, L. Caccavelli, N. Mahlaoui, V. Kiermer, D. Mittelstaedt, C. Bellesme, N. Lahlou, F. Lefrere, S. Blanche, M. Audit, E. Payen, P. Leboulch, B. l'Homme, P. Bougneres, C. Von Kalle, A. Fischer, M. Cavazzana-Calvo, P. Aubourg, Hematopoietic stem cell gene therapy with a lentiviral vector in X-linked adrenoleukodystrophy, Science 326 (5954) (2009) $818-823$.

[10] F. Eichler, C. Duncan, P.L. Musolino, P.J. Orchard, S. De Oliveira, A.J. Thrasher, M. Armant, C. Dansereau, T.C. Lund, W.P. Miller, G.V. Raymond, R. Sankar,
A.J. Shah, C. Sevin, H.B. Gaspar, P. Gissen, H. Amartino, D. Bratkovic, N.J.C. Smith, A.M. Paker, E. Shamir, T. O'Meara, D. Davidson, P. Aubourg, D.A. Williams, Hematopoietic stem-cell gene therapy for cerebral adrenoleukodystrophy, N. Engl. J. Med. 377 (17) (2017) 1630-1638.

[11] J. Berger, A. Pujol, P. Aubourg, S. Forss-Petter, Current and future pharmacological treatment strategies in X-linked adrenoleukodystrophy, Brain Pathol. 20 (4) (2010) $845-856$.

[12] N. Launay, C. Aguado, S. Fourcade, M. Ruiz, L. Grau, J. Riera, C. Guilera, M. Giros, I. Ferrer, E. Knecht, A. Pujol, Autophagy induction halts axonal degeneration in a mouse model of X-adrenoleukodystrophy, Acta Neuropathol. 129 (3) (2015) 399-415.

[13] N. Launay, M. Ruiz, L. Grau, F.J. Ortega, E.V. Ilieva, J.J. Martinez, E. Galea, I. Ferrer, E. Knecht, A. Pujol, S. Fourcade, Tauroursodeoxycholic bile acid arrests axonal degeneration by inhibiting the unfolded protein response in X-linked adrenoleukodystrophy, Acta Neuropathol. 133 (2) (2017) 283-301.

[14] L. Morato, J. Galino, M. Ruiz, N.Y. Calingasan, A.A. Starkov, M. Dumont, A. Naudi, J.J. Martinez, P. Aubourg, M. Portero-Otin, R. Pamplona, E. Galea, M.F. Beal, I. Ferrer, S. Fourcade, A. Pujol, Pioglitazone halts axonal degeneration in a mouse model of X-linked adrenoleukodystrophy, Brain 136 (Pt 8) (2013) 2432-2443.

[15] P. Ranea-Robles, N. Launay, M. Ruiz, N.Y. Calingasan, M. Dumont, A. Naudi, M. Portero-Otin, R. Pamplona, I. Ferrer, M.F. Beal, S. Fourcade, A. Pujol, Aberrant regulation of the GSK-3beta/NRF2 axis unveils a novel therapy for adrenoleukodystrophy, EMBO Mol. Med. 10 (8) (2018).

[16] A. Pujol, I. Ferrer, C. Camps, E. Metzger, C. Hindelang, N. Callizot, M. Ruiz, T. Pampols, M. Giros, J.L. Mandel, Functional overlap between ABCD1 (ALD) and ABCD2 (ALDR) transporters: a therapeutic target for X-adrenoleukodystrophy, Hum. Mol. Genet. 13 (23) (2004) 2997-3006.

[17] A. Pujol, C. Hindelang, N. Callizot, U. Bartsch, M. Schachner, J.L. Mandel, Late onset neurological phenotype of the X-ALD gene inactivation in mice: a mouse model for adrenomyeloneuropathy, Hum. Mol. Genet. 11 (5) (2002) 499-505.

[18] S. Fourcade, I. Ferrer, A. Pujol, Oxidative stress, mitochondrial and proteostasis malfunction in adrenoleukodystrophy: a paradigm for axonal degeneration, Free Radic. Biol. Med. 88 (Pt A) (2015) 18-29.

[19] S. Fourcade, J. Lopez-Erauskin, M. Ruiz, I. Ferrer, A. Pujol, Mitochondrial dysfunction and oxidative damage cooperatively fuel axonal degeneration in X-linked adrenoleukodystrophy, Biochimie 98 (2014) 143-149.

[20] J. Galino, M. Ruiz, S. Fourcade, A. Schluter, J. Lopez-Erauskin, C. Guilera, M. Jove, A. Naudi, E. Garcia-Arumi, A.L. Andreu, A.A. Starkov, R. Pamplona, I. Ferrer, M. Portero-Otin, A. Pujol, Oxidative damage compromises energy metabolism in the axonal degeneration mouse model of X-adrenoleukodystrophy, Antioxidants Redox Signal. 15 (8) (2011) 2095-2107.

[21] R. El Bejjani, M. Hammarlund, Neural regeneration in Caenorhabditis elegans, Annu. Rev. Genet. 46 (2012) 499-513.

[22] M.A. Larkin, G. Blackshields, N.P. Brown, R. Chenna, P.A. McGettigan, H. McWilliam, F. Valentin, I.M. Wallace, A. Wilm, R. Lopez, J.D. Thompson, T.J. Gibson, D.G. Higgins, Clustal W and clustal X version 2.0, Bioinformatics 23 (21) (2007) 2947-2948.

[23] C. Mello, A. Fire, DNA transformation, Methods Cell Biol. 48 (1995) 451-482.

[24] M. Finney, G. Ruvkun, The unc-86 gene product couples cell lineage and cell identity in C. elegans, Cell 63 (5) (1990) 895-905.

[25] J. Folch, M. Lees, G.H. Sloane Stanley, A simple method for the isolation and purification of total lipides from animal tissues, J. Biol. Chem. 226 (1) (1957) 497-509.

[26] C.T. Turgeon, A.B. Moser, L. Morkrid, M.J. Magera, D.K. Gavrilov, D. Oglesbee, K. Raymond, P. Rinaldo, D. Matern, S. Tortorelli, Streamlined determination of lysophosphatidylcholines in dried blood spots for newborn screening of X-linked adrenoleukodystrophy, Mol. Genet. Metabol. 114 (1) (2015) 46-50.

[27] H.J. Joo, Y.H. Yim, P.Y. Jeong, Y.X. Jin, J.E. Lee, H. Kim, S.K. Jeong, D.J. Chitwood, Y.K. Paik, Caenorhabditis elegans utilizes dauer pheromone biosynthesis to dispose of toxic peroxisomal fatty acids for cellular homoeostasis, Biochem. J. 422 (1) (2009) 61-71.

[28] J.N. Sleigh, S.D. Buckingham, B. Esmaeili, M. Viswanathan, E. Cuppen, B.M. Westlund, D.B. Sattelle, A novel Caenorhabditis elegans allele, smn-1(cb131), mimicking a mild form of spinal muscular atrophy, provides a convenient drug screening platform highlighting new and pre-approved compounds, Hum. Mol. Genet. 20 (2) (2010) 245-260.

[29] M.L. Toth, I. Melentijevic, L. Shah, A. Bhatia, K. Lu, A. Talwar, H. Naji, C. IbanezVentoso, P. Ghose, A. Jevince, J. Xue, L.A. Herndon, G. Bhanot, C. Rongo, D.H. Hall, M. Driscoll, Neurite sprouting and synapse deterioration in the aging Caenorhabditis elegans nervous system, J. Neurosci. 32 (26) (2012) 8778-8790.

[30] L.R. Earls, M.L. Hacker, J.D. Watson, D.M. Miller 3rd, Coenzyme Q protects Caenorhabditis elegans GABA neurons from calcium-dependent degeneration, Proc. Natl. Acad. Sci. U. S. A. 107 (32) (2010) 14460-14465.

[31] S.O. Zhang, A.C. Box, N. Xu, J. Le Men, J. Yu, F. Guo, R. Trimble, H.Y. Mak, Genetic and dietary regulation of lipid droplet expansion in Caenorhabditis elegans, Proc. Natl. Acad. Sci. U. S. A. 107 (10) (2010) 4640-4645.

[32] C.Y. Ewald, J.M. Hourihan, M.S. Bland, C. Obieglo, I. Katic, L.E. Moronetti Mazzeo, J. Alcedo, T.K. Blackwell, N.E. Hynes, NADPH oxidase-mediated redox signaling promotes oxidative stress resistance and longevity through memo-1 in C. elegans, eLife 6 (2017).

[33] W. Yang, S. Hekimi, A mitochondrial superoxide signal triggers increased longevity in Caenorhabditis elegans, PLoS Biol. 8 (12) (2010) e1000556.

[34] S.W. Smith, L.C.t. Latta, D.R. Denver, S. Estes, Endogenous ROS levels in C. elegans under exogenous stress support revision of oxidative stress theory of life-history tradeoffs, BMC Evol. Biol. 14 (2014) 161.

[35] P. Gonzalez-Cabo, A. Bolinches-Amoros, J. Cabello, S. Ros, S. Moreno, H.A. Baylis, F. Palau, R.P. Vazquez-Manrique, Disruption of the ATP-binding cassette B7 (ABTM-1/ABCB7) induces oxidative stress and premature cell death in Caenorhabditis elegans, J. Biol. Chem. 286 (24) (2011) 21304-21314.

[36] H. Ishiguro, K. Yasuda, N. Ishii, K. Ihara, T. Ohkubo, M. Hiyoshi, K. Ono, N. Senoo- 
Matsuda, O. Shinohara, F. Yosshii, M. Murakami, P.S. Hartman, M. Tsuda, Enhancement of oxidative damage to cultured cells and Caenorhabditis elegans by mitochondrial electron transport inhibitors, IUBMB Life 51 (4) (2001) 263-268.

[37] M.P. Murphy, R.A. Smith, Targeting antioxidants to mitochondria by conjugation to lipophilic cations, Annu. Rev. Pharmacol. Toxicol. 47 (2007) 629-656.

[38] L.F. Ng, J. Gruber, I.K. Cheah, C.K. Goo, W.F. Cheong, G. Shui, K.P. Sit, M.R. Wenk, B. Halliwell, The mitochondria-targeted antioxidant MitoQ extends lifespan and improves healthspan of a transgenic Caenorhabditis elegans model of Alzheimer disease, Free Radic. Biol. Med. 71 (2014) 390-401.

[39] S. Fourcade, M. Ruiz, C. Camps, A. Schluter, S.M. Houten, P.A. Mooyer, T. Pampols, G. Dacremont, R.J. Wanders, M. Giros, A. Pujol, A key role for the peroxisomal ABCD2 transporter in fatty acid homeostasis, Am. J. Physiol. Endocrinol. Metab. 296 (1) (2009) E211-E221.

[40] K.A. Cunningham, K. Ashrafi, Fat rationing in dauer times, Cell Metabol. 9 (2) (2009) 113-114.

[41] A. Schluter, A. Real-Chicharro, T. Gabaldon, F. Sanchez-Jimenez, A. Pujol, PeroxisomeDB 2.0: an integrative view of the global peroxisomal metabolome, Nucleic Acids Res. 38 (Database issue) (2010) D800-D805.

[42] Y. Fujiki, Y. Matsuzono, T. Matsuzaki, M. Fransen, Import of peroxisomal membrane proteins: the interplay of Pex3p- and Pex19p-mediated interactions, Biochim. Biophys. Acta 1763 (12) (2006) 1639-1646.

[43] S. Yokota, S.H. Togo, M. Maebuchi, M. Bun-Ya, C.M. Haraguchi, T. Kamiryo, Peroxisomes of the nematode Caenorhabditis elegans: distribution and morphological characteristics, Histochem. Cell Biol. 118 (4) (2002) 329-336.

[44] A.B. Moser, N. Kreiter, L. Bezman, S. Lu, G.V. Raymond, S. Naidu, H.W. Moser, Plasma very long chain fatty acids in 3,000 peroxisome disease patients and 29,000 controls, Ann. Neurol. 45 (1) (1999) 100-110.

[45] M.C. van de Beek, I.M. Dijkstra, S. Kemp, Method for measurement of peroxisomal very long-chain fatty acid beta-oxidation and de novo C26:0 synthesis activity in living cells using stable-isotope labeled docosanoic acid, Methods Mol. Biol. 1595 (2017) 45-54.

[46] C.W. van Roermund, L. Ijlst, T. Wagemans, R.J. Wanders, H.R. Waterham, A role for the human peroxisomal half-transporter ABCD3 in the oxidation of dicarboxylic acids, Biochim. Biophys. Acta 1841 (4) (2014) 563-568.

[47] S. Fourcade, J. Lopez-Erauskin, J. Galino, C. Duval, A. Naudi, M. Jove, S. Kemp, F. Villarroya, I. Ferrer, R. Pamplona, M. Portero-Otin, A. Pujol, Early oxidative damage underlying neurodegeneration in X-adrenoleukodystrophy, Hum. Mol. Genet. 17 (12) (2008) 1762-1773.

[48] N. Kruska, P. Schonfeld, A. Pujol, G. Reiser, Astrocytes and mitochondria from adrenoleukodystrophy protein (ABCD1)-deficient mice reveal that the adrenoleukodystrophy-associated very long-chain fatty acids target several cellular energydependent functions, Biochim. Biophys. Acta 1852 (5) (2015) 925-936.

[49] J. Lopez-Erauskin, S. Fourcade, J. Galino, M. Ruiz, A. Schluter, A. Naudi, M. Jove, M. Portero-Otin, R. Pamplona, I. Ferrer, A. Pujol, Antioxidants halt axonal degeneration in a mouse model of X-adrenoleukodystrophy, Ann. Neurol. 70 (1) (2011) 84-92.

[50] J. Lopez-Erauskin, J. Galino, M. Ruiz, J.M. Cuezva, I. Fabregat, D. Cacabelos, J. Boada, J. Martinez, I. Ferrer, R. Pamplona, F. Villarroya, M. Portero-Otin, S. Fourcade, A. Pujol, Impaired mitochondrial oxidative phosphorylation in the peroxisomal disease X-linked adrenoleukodystrophy, Hum. Mol. Genet. 22 (16) (2013) 3296-3305.

[51] L. Morato, M. Ruiz, J. Boada, N.Y. Calingasan, J. Galino, C. Guilera, M. Jove, A. Naudi, I. Ferrer, R. Pamplona, M. Serrano, M. Portero-Otin, M.F. Beal, S. Fourcade, A. Pujol, Activation of sirtuin 1 as therapy for the peroxisomal disease adrenoleukodystrophy, Cell Death Differ. 22 (11) (2015) 1742-1753.

[52] H. Cinar, S. Keles, Y. Jin, Expression profiling of GABAergic motor neurons in Caenorhabditis elegans, Curr. Biol. 15 (4) (2005) 340-346.

[53] D. Hoepfner, D. Schildknegt, I. Braakman, P. Philippsen, H.F. Tabak, Contribution of the endoplasmic reticulum to peroxisome formation, Cell 122 (1) (2005) 85-95.

[54] A. Schluter, S. Fourcade, R. Ripp, J.L. Mandel, O. Poch, A. Pujol, The evolutionary origin of peroxisomes: an ER-peroxisome connection, Mol. Biol. Evol. 23 (4) (2006) $838-845$.

[55] L.H. Wong, A.T. Gatta, T.P. Levine, Lipid transfer proteins: the lipid commute via shuttles, bridges and tubes, Nat. Rev. Mol. Cell Biol. 20 (2) (2019) 85-101.

[56] P.T. Bozza, J.P. Viola, Lipid droplets in inflammation and cancer, Prostaglandins Leukot. Essent. Fatty Acids 82 (4-6) (2010) 243-250.

[57] A.G. Cabodevilla, L. Sanchez-Caballero, E. Nintou, V.G. Boiadjieva, F. Picatoste, A. Gubern, E. Claro, Cell survival during complete nutrient deprivation depends on lipid droplet-fueled beta-oxidation of fatty acids, J. Biol. Chem. 288 (39) (2013) $27777-27788$.

[58] C.R. Santos, A. Schulze, Lipid metabolism in cancer, FEBS J. 279 (15) (2012) 2610-2623.

[59] H.Y. Mak, Lipid droplets as fat storage organelles in Caenorhabditis elegans: thematic review series: lipid droplet synthesis and metabolism: from yeast to man, $\mathrm{J}$ Lipid Res. 53 (1) (2012) 28-33.

[60] M. Artal-Sanz, N. Tavernarakis, Prohibitin couples diapause signalling to mitochondrial metabolism during ageing in C. elegans, Nature 461 (7265) (2009) 793-797.

[61] R. Zimmermann, J.G. Strauss, G. Haemmerle, G. Schoiswohl, R. BirnerGruenberger, M. Riederer, A. Lass, G. Neuberger, F. Eisenhaber, A. Hermetter, R. Zechner, Fat mobilization in adipose tissue is promoted by adipose triglyceride lipase, Science 306 (5700) (2004) 1383-1386.

[62] H.M. Cocheme, M.P. Murphy, Complex I is the major site of mitochondrial superoxide production by paraquat, J. Biol. Chem. 283 (4) (2008) 1786-1798.

[63] A. Heddi, G. Stepien, P.J. Benke, D.C. Wallace, Coordinate induction of energy gene expression in tissues of mitochondrial disease patients, J. Biol. Chem. 274 (33) (1999) 22968-22976.

[64] W.Y. Tsang, L.C. Sayles, L.I. Grad, D.B. Pilgrim, B.D. Lemire, Mitochondrial re spiratory chain deficiency in Caenorhabditis elegans results in developmental arrest and increased life span, J. Biol. Chem. 276 (34) (2001) 32240-32246.

[65] E. Galea, N. Launay, M. Portero-Otin, M. Ruiz, R. Pamplona, P. Aubourg, I. Ferrer, A. Pujol, Oxidative stress underlying axonal degeneration in adrenoleukodystrophy: a paradigm for multifactorial neurodegenerative diseases? Biochim. Biophys. Acta 1822 (9) (2012) 1475-1488.

[66] J.K. Ho, H. Moser, Y. Kishimoto, J.A. Hamilton, Interactions of a very long chain fatty acid with model membranes and serum albumin. Implications for the pathogenesis of adrenoleukodystrophy, J. Clin. Invest. 96 (3) (1995) 1455-1463.

[67] B.J. Snow, F.L. Rolfe, M.M. Lockhart, C.M. Frampton, J.D. O'Sullivan, V. Fung, R.A. Smith, M.P. Murphy, K.M. Taylor, A double-blind, placebo-controlled study to assess the mitochondria-targeted antioxidant MitoQ as a disease-modifying therapy in Parkinson's disease, Mov. Disord. 25 (11) (2010) 1670-1674.

[68] M.J. Rossman, J.R. Santos-Parker, C.A.C. Steward, N.Z. Bispham, L.M. Cuevas, H.L. Rosenberg, K.A. Woodward, M. Chonchol, R.A. Gioscia-Ryan, M.P. Murphy, D.R. Seals, Chronic supplementation with a mitochondrial antioxidant (MitoQ) improves vascular function in healthy older adults, Hypertension 71 (6) (2018) 1056-1063.

[69] X.F. Chen, M.X. Tian, R.Q. Sun, M.L. Zhang, L.S. Zhou, L. Jin, L.L. Chen, W.J. Zhou, K.L. Duan, Y.J. Chen, C. Gao, Z.L. Cheng, F. Wang, J.Y. Zhang, Y.P. Sun, H.X. Yu, Y.Z. Zhao, Y. Yang, W.R. Liu, Y.H. Shi, Y. Xiong, K.L. Guan, D. Ye, SIRT5 inhibits peroxisomal ACOX1 to prevent oxidative damage and is downregulated in liver cancer, EMBO Rep. 19 (5) (2018).

[70] Y. Sandlers, A.B. Moser, W.C. Hubbard, L.E. Kratz, R.O. Jones, G.V. Raymond, Combined extraction of acyl carnitines and 26:0 lysophosphatidylcholine from dried blood spots: prospective newborn screening for X-linked adrenoleukodystrophy, Mol. Genet. Metabol. 105 (3) (2012) 416-420.

[71] L.R. Strachan, T.J. Stevenson, B. Freshner, M.D. Keefe, D. Miranda Bowles, J.L. Bonkowsky, A zebrafish model of X-linked adrenoleukodystrophy recapitulates key disease features and demonstrates a developmental requirement for abcd1 in oligodendrocyte patterning and myelination, Hum. Mol. Genet. 26 (18) (2017) 3600-3614.

[72] M.C. McGuinness, J.F. Lu, H.P. Zhang, G.X. Dong, A.K. Heinzer, P.A. Watkins, J. Powers, K.D. Smith, Role of ALDP (ABCD1) and mitochondria in X-linked adrenoleukodystrophy, Mol. Cell Biol. 23 (2) (2003) 744-753.

[73] A.P. Bailey, G. Koster, C. Guillermier, E.M. Hirst, J.I. MacRae, C.P. Lechene, A.D. Postle, A.P. Gould, Antioxidant role for lipid droplets in a stem cell niche of Drosophila, Cell 163 (2) (2015) 340-353.

[74] R.W. Klemm, J.P. Norton, R.A. Cole, C.S. Li, S.H. Park, M.M. Crane, L. Li, D. Jin, A. Boye-Doe, T.Y. Liu, Y. Shibata, H. Lu, T.A. Rapoport, R.V. Farese Jr., C. Blackstone, Y. Guo, H.Y. Mak, A conserved role for atlastin GTPases in regulating lipid droplet size, Cell Rep. 3 (5) (2013) 1465-1475.

[75] B. Renvoise, B. Malone, M. Falgairolle, J. Munasinghe, J. Stadler, C. Sibilla, S.H. Park, C. Blackstone, Reep1 null mice reveal a converging role for hereditary spastic paraplegia proteins in lipid droplet regulation, Hum. Mol. Genet. 25 (23) (2016) 5111-5125.

[76] L. Hulshagen, O. Krysko, A. Bottelbergs, S. Huyghe, R. Klein, P.P. Van Veldhoven, P.P. De Deyn, R. D'Hooge, D. Hartmann, M. Baes, Absence of functional peroxisomes from mouse CNS causes dysmyelination and axon degeneration, J. Neurosci. 28 (15) (2008) 4015-4027.

[77] M.R. Van Gilst, H. Hadjivassiliou, K.R. Yamamoto, A Caenorhabditis elegans nutrient response system partially dependent on nuclear receptor NHR-49, Proc. Natl. Acad. Sci. U. S. A. 102 (38) (2005) 13496-13501.

[78] O. Ilkun, N. Wilde, J. Tuinei, K.M. Pires, Y. Zhu, H. Bugger, J. Soto, B. Wayment, C. Olsen, S.E. Litwin, E.D. Abel, Antioxidant treatment normalizes mitochondrial energetics and myocardial insulin sensitivity independently of changes in systemic metabolic homeostasis in a mouse model of the metabolic syndrome, J. Mol. Cell. Cardiol. 85 (2015) 104-116.

[79] L. Liu, K. Zhang, H. Sandoval, S. Yamamoto, M. Jaiswal, E. Sanz, Z. Li, J. Hui, B.H. Graham, A. Quintana, H.J. Bellen, Glial lipid droplets and ROS induced by mitochondrial defects promote neurodegeneration, Cell 160 (1-2) (2015) 177-190.

[80] D.M. Van Den Brink, A. Cubizolle, G. Chatelain, N. Davoust, V. Girard, S. Johansen, F. Napoletano, P. Dourlen, L. Guillou, C. Angebault-Prouteau, N. Bernoud-Hubac, M. Guichardant, P. Brabet, B. Mollereau, Physiological and pathological roles of FATP-mediated lipid droplets in Drosophila and mice retina, PLoS Genet. 14 (9) (2018) e1007627.

[81] C.M. Kassmann, C. Lappe-Siefke, M. Baes, B. Brugger, A. Mildner, H.B. Werner, O. Natt, T. Michaelis, M. Prinz, J. Frahm, K.A. Nave, Axonal loss and neuroinflammation caused by peroxisome-deficient oligodendrocytes, Nat. Genet. 39 (8) (2007) 969-976.

[82] G. Oikonomou, S. Shaham, The glia of Caenorhabditis elegans, Glia 59 (9) (2011) 1253-1263.

[83] Z. Shao, S. Watanabe, R. Christensen, E.M. Jorgensen, D.A. Colon-Ramos, Synapse location during growth depends on glia location, Cell 154 (2) (2013) 337-350.

[84] L.M. Kennedy, S.C. Pham, A. Grishok, Nonautonomous regulation of neuronal migration by insulin signaling, DAF-16/FOXO, and PAK-1, Cell Rep. 4 (5) (2013) 996-1009.

[85] A.L.A. Nichols, E. Meelkop, C. Linton, R. Giordano-Santini, R.K. Sullivan, A. Donato, C. Nolan, D.H. Hall, D. Xue, B. Neumann, M.A. Hilliard, The apoptotic engulfment machinery regulates axonal degeneration in C. elegans neurons, Cell Rep. 14 (7) (2016) 1673-1683. 